\title{
Joint Sensing and Power Allocation in Nonconvex Cognitive Radio Games: Quasi-Nash Equilibria
}

\author{
Jong-Shi Pang ${ }^{1}$ and Gesualdo Scutari ${ }^{2}$ \\ ${ }^{1}$ Dept. of Industrial and Enterprise Systems Engineering, University of Illinois, Urbana, IL 61801, U.S.A. \\ ${ }^{2}$ Dept. of Electrical Engineering, State University of New York at Buffalo, Buffalo, NY 14260, U.S.A. \\ Emails:jspang@illinois.edu and gesualdo@buffalo.edu.*
}

Submitted to IEEE Transactions on Signal Processing, April 7, 2012. Second revision, Dec. 25, 2012.

\begin{abstract}
In this paper, we propose a novel class of Nash problems for Cognitive Radio (CR) networks composed of multiple primary users (PUs) and secondary users (SUs) wherein each SU (player) competes against the others to maximize his own opportunistic throughput by choosing jointly the sensing duration, the detection thresholds, and the vector power allocation over a multichannel link. In addition to power budget constraints, several (deterministic or probabilistic) interference constraints can be accommodated in the proposed general formulation, such as constraints on the maximum individual/aggregate (probabilistic) interference tolerable from the PUs. To keep the optimization as decentralized as possible, global interference constraints, when present, are imposed via pricing; the prices are thus additional variables to be optimized. The resulting players' optimization problems are nonconvex and there are price clearance conditions associated with the nonconvex global interference constraints to be satisfied by the equilibria of the game, which make the analysis of the proposed game a challenging task; none of classical results in the game theory literature can be successfully applied. To deal with the nonconvexity of the game, we introduce a relaxed equilibrium concept-the Quasi-Nash Equilibrium (QNE) -and study its main properties, performance, and connection with local Nash equilibria. Quite interestingly, the proposed game theoretical formulations yield a considerable performance improvement with respect to current centralized and decentralized designs of CR systems, which validates the concept of QNE.
\end{abstract}

\section{Introduction and Motivation}

In the last decade, Cognitive Radio (CR) has received considerable attention as a way to improve the efficiency of radio networks [1, 2]. The CR networks adopt a hierarchical access structure where the primary users (PUs) are the legacy spectrum holders while the secondary users (SUs) are the unlicensed users who sense the electromagnetic environment and adapt their transceiver parameters as well as the resource allocation decisions in order to dynamically access temporally unoccupied spectrum regions.

The challenge for a reliable sensing algorithm is to identify suitable transmission opportunities without compromising the integrity of the PUs. One of the design criteria is to make the probability of false alarm

*The work of Pang is based on research supported by the U.S.A. National Science Foundation grant CMMI 0969600 and by the Air Force Office of Sponsored Research award No. FA9550-09-10329. The work of Scutari was supported by U.S.A. National Science Foundation grant CSM 1218717.

Copyright (c) 2012 IEEE. Personal use of this material is permitted. However, permission to use this material for any other purposes must be obtained from the IEEE by sending a request to pubs-permissions@ieee.org. 
as low as possible, since it measures the percentage of vacant spectrum which is misclassified as busy, increasing thus the opportunistic usage of the spectrum from the SUs. On the other hand, in order to limit the probability of interfering with PUs, it is desirable to keep the missed detection probability as low as possible. The detection thresholds are the trade-off factor between the false alarm and the missed detection probabilities; low thresholds will result in high false alarm rate in favor of low missed detection probability and vice versa. Alternatively, the choice of the sensing time offers a trade-off between the quality and speed of sensing: increasing the sensing times permits to reach both low false alarm and detection probability values, thus reducing the time available for secondary transmissions, which would result in low SUs' throughput. The above trade-off calls naturally for a joint determination of the sensing and transmission parameters of the SUs, under the paradigm of selfish behavior among these users. The modeling and analysis of this competitive multi-agent optimization is the main subject of this work.

\subsection{Related work}

The joint optimization of the sensing and transmission parameters has been only partially addressed in the literature; current works on the subject can be divided in the following three main classes.

- Optimization of the sensing parameters only [3, 4, 5, 6, 7, 8,. In [3, 4, the authors proposed alternative centralized schemes that optimize the detection thresholds for a bank of energy detectors, in order to maximize the so-called opportunistic throughput, while keeping the sensing time and the transmission parameters of the SU fixed and given a priori. The optimization of the sensing time and the sensing time/detection thresholds for a given missed detection probability and transmission rate of one SU was addressed in [5] and [7, 8, respectively. A throughput-sensing trade-off for a given transmission rate of the SU was studied in [6]. In the above papers there is no optimization of the SU's transmission strategies, and the proposed schemes are applicable only to CR scenarios composed by one PU and SU.

- Optimization of the transmission parameters only [9, 10, 11, 12, 13, 14, 15, 16, 17, 18. These papers address the optimization of the SUs' transceivers in a multiuser OFDM CR scenario; several centralized [9, 10, 11, 18] schemes or distributed algorithms based on a game theoretic approach [12, 13, 14, 15] were proposed; a recent overview of the latter approaches can be found in [15, 19]. A general framework based on variational inequalities was proposed in [16, 17] to study and solve in a distributed way convex noncooperative games with (possibly) side (i.e., coupling) constraints (e.g., interference constraints). In all the aforementioned papers the sensing process is not considered as part of the optimization; in fact the SUs do not perform any sensing but they are allowed to transmit over the licensed spectrum provided that they satisfy interference constraints imposed by the PUs, no matter if the PUs are active of not.

- Joint optimization of some of the sensing/transmission parameters [20, 21, 22, 23, 24, 25]. In [20] (or [21, 22]), the sensing time and the transmit power (or the power allocation [21, 22] over multichannel links) of one SU were jointly optimized while keeping the detection probability (and thus the decision threshold) fixed to a target value. In [23, 24], the authors focused on the joint optimization of the power allocation and the equi-false alarm rate of one SU [23] over multi-channel links, for a fixed sensing time. The case of multiple SUs and one PU was considered in [24] (and more recently in [25]), under the same assumptions of [23]; however no formal analysis of the proposed formulation was provided. Moreover, 
these papers [23, 24, 25] did not consider the sensing overhead as part of the optimization, leaving unclear how to optimally choose the sensing time.

\subsection{Main contributions}

What emerges from the analysis of existing literature is that, up to now, state-of-the-art approaches have focused on the optimization of specific features and single components of a CR system in isolation. In this paper we move a step ahead and propose a novel class of Nash Equilibrium Problems (NEPs), suitable for designing multiple primary/secondary user CR networks, under different practical settings. Aiming to dynamically access temporally unoccupied primary spectrum regions, in our formulation, the SUs maximize their own opportunistic throughput by jointly optimizing the sensing time, the decision thresholds of a bank of energy detectors, and the power allocation over multi-channel links. Because of sensing errors, it may happen however that the SUs attempt to access part of licensed spectrum that is still occupied by active PUs, causing thus harmful interference. This motivates the introduction of probabilistic (rather than deterministic) interference constraints that are imposed to control the power radiated over the licensed spectrum only when a missed detection event occurs (in a probabilistic sense). The proposed formulation accommodates alternative combinations of power/interference constraints. For instance, in addition to classical (deterministic) transmit power (and possibly spectral masks) constraints, we envisage the use of (probabilistic) average individual (i.e., on each SU) and/or global (i.e., over all the SUs) interference tolerable by the primary receivers. Local interference constraints fit naturally to scenarios where the SUs are not willing to cooperate; whereas the global ones, which are less conservative, are more suitable for settings where SUs may want to trade some signaling for better performance. By

imposing a coupling among the power allocations of the SUs, global interference constraints introduce a new challenge in the system design: how to enforce global interference constraints without requiring a centralized optimization? A major contribution of the paper is to address this issue by introducing a pricing mechanism in the game, through a penalization in the players' objective functions. The prices will be then additional variables to be optimized, while guaranteeing the global interference constraints to be satisfied at any solution of the game.

The resulting game-theoretical formulations belong to the class of nonconvex games, where the nonconvexity occurs at both the objective functions and feasible sets of the SUs' optimization problems. Indeed the objective functions of the SUs (to be maximized) are not quasi-concave and the local/global interference constraints are bi-convex and thus nonconvex; moreover, in the presence of global interference constraints, there are possibly unbounded price variables to be optimized and pricing clearing conditions associated with these constraints to be satisfied at the equilibrium. All this makes the analysis of the proposed games a challenging task; none of previous results in the game theory literature can be successfully applied (see Sec. 4 for more details); without (quasi-)concavity of the players' payoff functions or convexity of the strategy sets, a solution of the game-the Nash Equilibrium (NE)-may not even exist [26, 27]. To overcome this issue, alternative refinements of the NE concept have been introduced in the literature, in the form of "local" solutions (see, e.g., [26, 28, 29, 30] and references therein): in contrast to the NE that is resistant to "arbitrarily large" deviations of single player's strategies, local equilibria require 
stability only against "small" unilaterally deviations of the players. Among all, we mention here some local solution concepts that have been proposed in the literature: such as the "critical NE" [26], the "Local NE" (LNE) [29], and the "generalized equilibrium" [30]. Despite their theoretical interest, those local solution concepts have a very limited applicability: when available, there are only abstract mathematical conditions granting their existence (see, e.g., [26, 30]), whose verification for games arising from realistic applications such as those presented in the present paper remains elusive. A system design based on these concepts may lead to an unpredictable system behavior (e.g., equilibrium existence vs. nonexistence, convergence of algorithms vs. nonconvergence), which is not a desirable feature in practice.

In this paper, we propose an alternative formalization of solution in nonconvex games; building on the well-established and accepted definition of stationary solutions of a nonlinear programming, we introduce a (relaxed) equilibrium concept for a nonconvex NEP, which is a solution of the aggregated stationarity conditions (the Karush-Kuhn-Tucker conditions) of the players' optimization problems. We call such a stationary tuple a quasi-Nash equilibrium (QNE) of the (nonconvex) game; the prefix "quasi" is intended to signify that a NE (if it exists) must be a QNE under mild conditions (constraint qualifications). While the concept of QNE seems quite natural and conceptually similar to the idea of critical NE [26] and generalized equilibrium [30, its analysis is not an easy task (cf. Sec.4), and cannot be addressed using results in previous papers (e.g., [26, 30]); for instance, in the absence of convexity or boundedness of the price variables that could lead to the non-existence of a NE, the existence of a QNE is by no means obvious. Building on our recent results in [31, a major contribution of the paper is to provide a satisfactory characterization of the QNE, in terms of existence, connection with the (L)NE, and performance. The main features of the QNE are the following: i) it always exists for the proposed class of nonconvex games; ii) it is shown to have some local optimality properties (in the sense described in Sec. 6); and iii) the proposed joint optimization of sensing and transmission strategies based on the QNE yields a considerable performance improvements (more than one hundred per cent in "high" interference regimes) with respect to both centralized [18] and decentralized [12, 14, 15, 17] CR designs that do not perform any joint optimization. These desired properties validate the use of the QNE both theoretically and practically.

At the QNE of the game, the optimal sensing times of the SUs tend to be different, implying that some SUs may start transmitting while some others are still in the sensing phase, which may introduce a significant performance degradation in the sensing process of the other SUs. To overcome this issue, a further contribution of the paper is to propose a distributed approach to "force" (at least asymptotically) the same optimal sensing time for all the SUs; which is referred to as the equi-sensing case.

In summary, the main contributions of the paper are the following:

- We propose a novel class of NEPs (possibly with pricing), where for the first time a joint optimization of the detection threshold, the sensing time, and the transmission strategies of multiple SUs is performed, under power and several (novel) local and/or global probabilistic interference constraints;

- We introduce a relaxed equilibrium concept-the QNE-and develop a novel optimization-based theory for studying its main properties, the connection with the LNE, and its performance;

- We show how to modify the original NEPs to impose in a distributed way an optimal equi-sensing time to all the SUs, and study the main properties of the resulting game. 
Overall, we introduce a new line of analysis based on Variational Inequalities (VIs) in the literature of nonconvex games (possibly) with pricing that is expected to be broadly applicable for other game models.

Because of the space limitation, we omit here details on how to solve the proposed games, which is the subject of a companion paper [32, where we propose alternative distributed algorithms along with their convergence properties and a detailed discussion on their practical implementation; and also establish the existence of NE of the games under a set of small-interference assumptions between the SUs and PUs (that are not needed for the QNE).

The rest of the paper is organized as follows. Sec. 2 describes the system model, whereas Sec. 3 introduces the new game-theoretical formulations. The analysis of the game is addressed in Sec.4, where the LNE and the concept of QNE are introduced, and their properties and connection are studied. Sec. 5 focuses on the equi-sensing case; whereas Sec.6 outlines some extensions of the proposed formulations covering more general settings. Sec.7 provides some numerical results showing the quality of the proposed approach; and finally Sec. 8 draws the conclusions.

\section{System Model}

We consider a scenario composed of $Q$ active SUs, each formed by a transmitter-receiver pair, coexisting in the same area and sharing the same band. We focus on block transmissions over SISO frequency-selective channels. It is well known that, in such a case, multicarrier transmission is capacity achieving for large block-length. Based on the 802.22-Wireless-Regional-Area-Networks standard (WRAN) [33], we assume that the Medium Access Control (MAC) frame is divided in two slots: one sensing slot and one data slot. During the sensing interval, the SUs stay silent and sense the electromagnetic environment looking for the spectrum holes, whereas during the data slot they transmit (possibly) simultaneously over the portions of the licensed spectrum detected as available. The sensing and transmission phases are described in Sec. 2.1 and Sec. 2.2. respectively, whereas the proposed joint optimization of the sensing and transmission strategies over the two phases is introduced in Sec. 3.

We wish to point out that, even if we focus on ad-hoc networks and adopt the classical CR terminology

(e.g., PUs, SUs, etc.), the proposed model and results are applicable to broader scenarios, wherever there are heterogeneous (small-cell) networks organized in a hierarchical or multi-tier structure and sharing the same spectrum (e.g., due to the spatial reuse of the frequencies). This happens for example in femtocell networks, where femto access points (FAPs) serve femto users, subject to some constraints on the interference radiated toward mobile users served by macro base stations.

\subsection{The spectrum sensing problem}

For notational simplicity, we preliminarily introduce the sensing problem in a CR scenario composed of one active PU and under some simplified assumptions; a more general setting (e.g., multiple active PUs) is addressed in Sec. [6 within the framework of robust detection.

The spectrum sensing problem of $\mathrm{SU} q=1, \ldots, Q$ on subcarrier $k=1, \ldots, N$ is formulated as a binary hypothesis testing, with the following two sets of hypotheses: at time index $n=1,2, \ldots, K_{q}$, 


$$
\begin{array}{lll}
\text { (PU signal absent) } & \mathcal{H}_{k \mid 0}: & y_{q, k}[n]=w_{q, k}[n] \\
\text { (PU signal present) } & \mathcal{H}_{k \mid 1}: & y_{q, k}[n]=I_{q, k}[n]+w_{q, k}[n],
\end{array}
$$

where $\mathcal{H}_{k \mid 0}$ represents the absence of any primary signal over the subcarrier $k$-the received baseband complex signal $y_{q, k}[n]$ contains only additive background noise $w_{q, k}[n]$-and $\mathcal{H}_{k \mid 1}$ represents the presence of (at least) one PU-the received signal contains the primary signaling $I_{q, k}[n]$ corrupted by noise-and $K_{q}=\left\lfloor\tau_{q} f_{q}\right\rfloor \simeq \tau_{q} f_{q}$ is the number of samples, with $\tau_{q}$ and $f_{q}$ denoting the sensing time and the sampling frequency, respectively.

Given the hypothesis testing problem in (10), we make the following standard assumptions in the literature of sensing algorithms [3, 4, 5, 6, 7, 8, 20, 21, 23]: (A1) the noise $w_{q, k}[n]$ is drawn by a circularly symmetric complex stationary (ergodic) Gaussian process with zero mean and variance $\sigma_{q, k}^{2} ;(A 2)$ the primary signaling $I_{q, k}[n]$ are samples of a circularly symmetric complex stationary (ergodic) random process with zero mean, variance $\sigma_{I_{q, k}}^{2}$, and statistically independent of the noise process; $(A 3)$ the sample frequency $f_{q}$ is chosen so that the random variables (RVs) $\left\{y_{q, k}[n]\right\}_{n}$ are independent, for all $q$ and $k 1$ (A4) the system parameters-the spectrum occupancy status of the PUs and the primary/secondary (cross-)channels-change sufficiently slowly such that they can be assumed to be constant over each sensing/transmission interval; and $(A 5)$ the noise variance $\sigma_{q, k}^{2}$ and the power $\sigma_{I_{q, k}}^{2}$ of the primary signaling are estimate with no errors at the secondary receiver $q$. Note that $(A 5)$ can be relaxed by taking explicitly into account estimation errors in the knowledge of $\sigma_{q, k}^{2}$ and $\sigma_{I_{q, k}}^{2}$ as well as the effect of shadowing and fading, as outlined in Sec. 6. see also [35].

Within the Neyman-Pearson framework, the test statistic of SU $q$ over subcarrier $k$ based on the energy detector maximizing the detection probability under a given false alarm rate is [36]:

$$
D\left(\mathbf{Y}_{q, k}\right) \triangleq \frac{1}{K_{q}} \sum_{n=1}^{K_{q}}\left|y_{q, k}[n]\right|^{2} \underset{\mathcal{H}_{k \mid 0}}{\stackrel{\mathcal{H}_{k \mid 1}}{\gtrless}} \gamma_{q, k}
$$

where $\gamma_{q, k}$ is the decision threshold of $\mathrm{SU} q$ for the carrier $k=1, \ldots, N$, to be chosen to meet the required false alarm rate. Note that if the received samples can be assumed Gaussian distributed (and uncorrelated), the above energy-based decision is optimal; otherwise it is still a valuable choice when no a-priori information is available on the primary signal features. Moreover, it is interesting to report some results in [37, 38], where it has been argued that for several models, if the probability density functions under both hypotheses are perfectly known, energy detection performs close to the optimal detector. The case of partial knowledge of the probability density functions is discussed in Sec. 6 within the context of robust detection; see also 35 .

Invoking the central limit theorem, the random variable $D\left(\mathbf{Y}_{q, k}\right)$ can be approximated for sufficiently large $K_{q}=\tau_{q} f_{q}$ by a Gaussian distribution: for $i=1,2, D\left(\mathbf{Y}_{q, k}\right) \mid \mathcal{H}_{k \mid i} \sim \mathcal{N}\left(\mu_{q, k \mid i}, \sigma_{q, k \mid i}^{2} / K_{q}\right)$, where

$$
\mu_{q, k \mid i} \triangleq\left\{\begin{array} { l l } 
{ \sigma _ { q , k } ^ { 2 } , } & { \text { if } i = 0 , } \\
{ \sigma _ { I _ { q , k } } ^ { 2 } + \sigma _ { q , k } ^ { 2 } , } & { \text { if } i = 1 , }
\end{array} \quad \text { and } \quad \sigma _ { q , k | i } ^ { 2 } \triangleq \left\{\begin{array}{ll}
\sigma_{q, k}^{4}, & \text { if } i=0 \\
\mathrm{E}\left|I_{q, k}\right|^{4}+2 \sigma_{q, k}^{2}-\left(\sigma_{I_{q, k}}^{2}-\sigma_{q, k}^{2}\right)^{2}, & \text { if } i=1 .
\end{array}\right.\right.
$$

\footnotetext{
${ }^{1}$ We can relax the constraint on $f_{q}$ by requiring in (A3) instead block independence of the RVs $\left\{y_{q, k}[n]\right\}_{n}$, and invoke for our derivations the central limit theorem valid for correlated ( $m$-indepedend) RVs, as stated, e.g., in [34, Th. 2.8.1]; because of the space limitation, we omit these details here and stay within the original assumption (A3).
} 
An explicit expression of the expected value $\mathrm{E}\left|I_{q, k}\right|^{4}$ above depends on the PUs' signaling features. For example, if the primary signals are drawn from a PSK modulation, then $\sigma_{q, k \mid 1}^{2}=\sigma_{q, k}^{2}\left(2 \sigma_{I_{q, k}}^{2}+\sigma_{q, k}^{2}\right)[3$; whereas if the PU signaling is assumed to be Gaussian, then $\sigma_{q, k \mid 1}^{2}=\left(\sigma_{I_{q, k}}^{2}+\sigma_{q, k}^{2}\right)^{2}[5]$.

The performance of the energy detector scheme are measured in terms of the detection probability $P_{q, k}^{\mathrm{d}}\left(\gamma_{q, k}, \tau_{q}\right) \triangleq \operatorname{Prob}\left\{D\left(\mathbf{Y}_{q, k}\right)>\gamma_{q, k} \mid \mathcal{H}_{1, k}\right\}$ and false alarm probability $P_{q, k}^{\mathrm{fa}}\left(\gamma_{q, k}, \tau_{q}\right) \triangleq \operatorname{Prob}\left\{D\left(\mathbf{Y}_{q, k}\right)>\right.$ $\left.\gamma_{q, k} \mid \mathcal{H}_{0, k}\right\}$ that, under the assumptions above, are given by

$$
P_{q, k}^{\mathrm{fa}}\left(\gamma_{q, k}, \tau_{q}\right)=\mathcal{Q}\left(\sqrt{\tau_{q} f_{q}} \frac{\gamma_{q, k}-\mu_{q, k \mid 0}}{\sigma_{q, k \mid 0}}\right) \text { and } P_{q, k}^{\mathrm{d}}\left(\gamma_{q, k}, \tau_{q}\right)=\mathcal{Q}\left(\sqrt{\tau_{q} f_{q}} \frac{\gamma_{q, k}-\mu_{q, k \mid 1}}{\sigma_{q, k \mid 1}}\right)
$$

where $\mathcal{Q}(x) \triangleq(1 / \sqrt{2 \pi}) \int_{x}^{\infty} e^{-t^{2} / 2} d t$ is the Q-function. The interpretation of $P_{q, k}^{\mathrm{fa}}\left(\gamma_{q, k}, \tau_{q}\right)$ and $P_{q, k}^{\mathrm{d}}\left(\gamma_{q, k}, \tau_{q}\right)$ within the CR scenario is the following: $1-P_{q, k}^{\text {fa }}$ signifies the probability of successfully identifying from the $\mathrm{SU} q$ a spectral hole over carrier $k$, whereas the missed detection probability $P_{q, k}^{\text {miss }} \triangleq 1-P_{q, k}^{\mathrm{d}}$ represents the probability of SU $q$ failing to detect the presence of the PUs on the subchannel $k$ and thus generating interference against the PUs. The free variables to optimize are the detection thresholds $\gamma_{q, k}$ 's and the sensing times $\tau_{q}$ 's; ideally, we would like to choose $\gamma_{q, k}$ 's and $\tau_{q}$ 's in order to minimize both $P_{q, k}^{\text {fa }}$ and $P_{q, k}^{\text {miss }}$, but (3) shows that there exists a trade-off between these two quantities that will affect both primary and secondary performance. It turns out that, $\gamma_{q, k}$ 's and $\tau_{q}$ 's can not be chosen by focusing only on the detection problem (as in classical decision theory), but the optimal choice of $\gamma_{q, k}$ and $\tau_{q}$ must be the result of a joint optimization of the sensing and transmission strategies over the two phases; such a novel optimization is formulated in Sec. 3.

\subsection{The transmission phase}

We model the set of the $Q$ active SUs as a frequency-selective $N$-parallel interference channel, where $N$ is the number of available subcarriers; no interference cancellation is performed at the secondary receivers and the Multi-User Interference (MUI) is treated as additive colored noise at each receiver. This model is suitable for most of the CR scenarios, where the SUs coexisting in the network operate in a uncoordinated manner without cooperating with each other, and no centralized authority is assumed to handle the network access for the SUs. The transmission strategy of each SU $q$ is then the power allocation vector $\mathbf{p}_{q}=\left\{p_{q, k}\right\}_{k=1}^{N}$ over the $N$ subcarriers $\left(p_{q, k}\right.$ is the power allocated over carrier $k$ ), subject to the following transmit power constraints

$$
\mathcal{P}_{q} \triangleq\left\{\mathbf{p}_{q} \in \mathbb{R}^{N}: \sum_{k=1}^{N} p_{q, k} \leq P_{q}, \quad 0 \leq \mathbf{p}_{q} \leq \mathbf{p}_{q}^{\max }\right\},
$$

where we also included (possibly) local spectral mask constraints $\mathbf{p}_{q}^{\max }=\left(p_{q, k}^{\max }\right)_{k=1}^{N}$ [the vector inequality in (44) is component-wise].

Opportunistic throughput. The goal of each SU is to optimize his "opportunistic spectral utilization" of the licensed spectrum, having no a priori knowledge of the probabilities on the PUs' presence. According to this paradigm, each subcarrier $k$ is available for the transmission of $\mathrm{SU} q$ if no primary signal is detected over that frequency band, which happens with probability $1-P_{q, k}^{\mathrm{fa}}$. This motivates the use of the aggregate 
opportunistic throughput as a measure of the spectrum efficiency of each SU q. Given the power allocation profile $\mathbf{p} \triangleq\left(\mathbf{p}_{q}\right)_{q=1}^{Q}$ of the SUs, the detection thresholds $\gamma_{q} \triangleq\left(\gamma_{q, k}\right)_{k=1}^{N}$, and sensing time $\tau_{q}$, the aggregate opportunistic throughput of $\mathrm{SU} q$ is defined as

$$
R_{q}\left(\tau_{q}, \mathbf{p}, \gamma_{q}\right)=\left(1-\frac{\tau_{q}}{T}\right) \sum_{k=1}^{N}\left[1-P_{q, k}^{\mathrm{fa}}\left(\gamma_{q, k}, \tau_{q}\right)\right] r_{q, k}(\mathbf{p})
$$

where $1-\tau_{q} / T\left(\tau_{q} \leq T\right)$ is the portion of the frame duration available for opportunistic transmissions, $P_{q, k}^{\mathrm{fa}}\left(\gamma_{q, k}, \tau_{q}\right)$ is the worst-case false alarm rate defined in (3) , and $r_{q, k}(\mathbf{p})$ is the maximum information rate achievable on secondary link $q$ over carrier $k$ when no primary signal is detected and the power allocation of the SUs is p. Under basic information theoretical assumptions, the maximum achievable rate $r_{q, k}(\mathbf{p})$ for a specific power allocation profile $p_{1, k}, \ldots, p_{Q, k}$ is

$$
r_{q, k}(\mathbf{p})=\log \left(1+\frac{\left|H_{q q}(k)\right|^{2} p_{q, k}}{\sigma_{q, k}^{2}+\sum_{r \neq q}\left|H_{r q}(k)\right|^{2} p_{r, k}}\right),
$$

where $\left\{H_{q q}(k)\right\}_{k=1}^{N}$ is the channel transfer function of the direct link $q$ and $\left\{H_{r q}(k)\right\}_{k=1}^{N}$ is the crosschannel transfer function between the secondary transmitter $r$ and the secondary receiver $q$; and $\sigma_{q, k}^{2}$ is the variance of the background noise over carrier $k$ at the receiver $q$ (assumed to be Gaussian zero-mean distributed).

Probabilistic interference constraints. Due to the inherent trade-off between $P_{q, k}^{\mathrm{fa}}$ and $P_{q, k}^{\text {miss }}$ (cf. Sec. 2.1), maximizing the aggregate opportunistic throughput (5) of SUs will result in low $P_{q, k}^{\mathrm{fa}}$ and thus large $P_{q, k}^{\text {miss }}$, hence causing harmful interference to PUs (which happens with probability $P_{q, k}^{\text {miss }}$ ). To control the interference radiated by the SUs, we propose to impose probabilistic interference constraints in the form of individual and/or global constraints. Individual interference constraints are imposed at the level of each SU $q$ to limit the average interference generated at the primary receiver, whereas global interference constraints limit the average aggregate interference generated by all the SUs, which is in fact the average interference experimented by the PU. Examples of such constraints are the following.

- Individual interference constraints:

$$
\sum_{k=1}^{N} P_{q, k}^{\operatorname{miss}}\left(\gamma_{q, k}, \tau_{q}\right) \cdot w_{q, k} \cdot p_{q, k} \leq I_{q}^{\max },
$$

- Global interference constraints,

$$
\sum_{q=1}^{Q} \sum_{k=1}^{N} P_{q, k}^{\operatorname{miss}}\left(\gamma_{q, k}, \tau_{q}\right) \cdot w_{q, k} \cdot p_{q, k} \leq I^{\max }
$$

where $I_{q}^{\max }\left[\right.$ or $I^{\max }$ ] is the maximum average interference allowed to be generated by the SU $q$ [or all the SU's], and $w_{q k}$ 's are a given set of positive weights. If an estimate of the cross-channel transfer functions $\left\{G_{q}(k)\right\}_{k=1}^{N}$ between the secondary transmitters $q$ 's and the primary receiver is available, then the natural choice of $w_{q k}$ is $w_{q k}=\left|G_{q}(k)\right|^{2}$, so that (7) and (8) become the average interference experienced at the primary receiver. In some scenarios where the primary receivers have a fixed geographical location, it may 
be possible to install some monitoring devices close to each primary receiver having the functionality of (cross-)channel measurement and estimation of $I_{q}^{\max }$ 's. In scenarios where this option is not feasible and the channel state information cannot be acquired, a different choice of the weights coefficients $w_{q, k}$ 's and the interference threshold $I_{q}^{\max }$ in (7) and (8) can be made, based e.g. on worst-case channel/interference statistics; several alternative options are discussed in our companion paper [32, which is devoted to the design of distributed solution algorithms along with their practical implementation.

Remark 1 (Individual vs. global constraints). The proposed individual and/or global interference constraints provide enough flexibility to explore the interplay between performance and signaling among the SUs, making thus the proposed model applicable to different CR scenarios. For instance, in the settings where the SUs cannot exchange any signaling, the system design based on individual interference constraints seems to be the most natural formulation (see Sec. 3.1); this indeed leads to totally distributed algorithms with no signaling among the SUs, as we show in the companion paper [32. On the other hand, there are scenarios where the SUs may want to trade some signaling for better performance; in such cases imposing global interference constraints rather than the more conservative individual constraints is expected to provide larger SUs' throughputs. This however introduces a new challenge: how to enforce global interference constraints in a distributed way? By imposing a coupling among the power allocations of all the SUs, global interference constraints in principle would call for a centralized optimization. A major contribution of the paper is to propose a pricing mechanism based on the relaxation of the coupling interference constraints as penalty term in the SUs' objective functions (see Sec. 3.2). In the companion paper [32], we show that this formulation leads to (fairly) distributed best-response algorithms where the SUs can update the prices via consensus based-schemes, which requires a limited signaling among the SUs, in favor of better performance.

\section{Joint Optimization of Sensing and Transmissions via Game Theory}

We focus now on the system design and formulate the joint optimization of the sensing parameters and the power allocation of the SUs within the framework of game theory. Motivated by the discussion in the previous section (cf. Remark 1), we propose next two classes of equilibrium problems: i) games with individual constraints only; and ii) games with individual and global constraints. The former formulation is suitable for modeling scenarios where the SUs are selfish users who are not willing to cooperate, whereas the latter class of games is applicable to the design of systems where the SUs can exchange limited signaling in favor of better performance. At the best of our knowledge, both formulations based on the joint optimization of sensing and transmission strategies are novel in the literature. We introduce first the two formulations in Sec. 3.1 and Sec. 3.2 below, and then provide a unified analysis of both games.

\subsection{Game with individual constraints}

In the proposed game, each $\mathrm{SU}$ is modeled as a player who aims to maximize his own opportunistic throughput $R_{q}\left(\mathbf{p}, \gamma_{q}, \tau_{q}\right)$ by choosing jointly a proper power allocation strategy $\mathbf{p}_{q}=\left(p_{q, k}\right)_{k=1}^{N}$, detection

thresholds $\gamma_{q} \triangleq\left(\gamma_{q, k}\right)_{k=1}^{N}$, and sensing time $\tau_{q}$, subject to power and individual probabilistic interference 
constraints. Stated in mathematical terms, player q's optimization problem is to determine, for given $\mathbf{p}_{-q} \triangleq\left(\left(p_{r, k}\right)_{k=1}^{N}\right)_{q \neq r=1}^{Q}$, a tuple $\left(\tau_{q}, \mathbf{p}_{q}, \gamma_{q}\right)$ such that

$$
\begin{array}{ll}
\underset{\begin{array}{c}
\tau_{q}, \mathbf{p}_{q}, \gamma_{q} \\
\text { subject to }
\end{array}}{\operatorname{maximize}} & R_{q}\left(\tau_{q}, \mathbf{p}, \gamma_{q}\right) \\
\text { (a) } & \sum_{k=1}^{N} P_{q, k}^{\operatorname{miss}}\left(\gamma_{q, k}, \tau_{q}\right) \cdot w_{q, k} \cdot p_{q, k} \leq I_{q}^{\max }, \\
\text { (b) } & P_{q, k}^{\mathrm{fa}}\left(\gamma_{q, k}, \tau_{q}\right) \leq \beta_{q, k}, \quad \text { and } \quad P_{q, k}^{\operatorname{miss}}\left(\gamma_{q, k}, \tau_{q}\right) \leq \alpha_{q, k}, \quad \forall k=1, \cdots, N, \\
\text { (c) } & \mathbf{p}_{q} \in \mathcal{P}_{q} \text { and } \tau_{q}^{\min } \leq \tau_{q} \leq \tau_{q}^{\max } .
\end{array}
$$

In (9) we also included additional lower and upper bounds of $\tau_{q}$ satisfying $0<\tau_{q}^{\min }<\tau_{q}^{\max }<T_{q}$ and upper bounds on detection and missed detection probabilities $0<\alpha_{q, k} \leq 1 / 2$ and $0<\beta_{q, k} \leq 1 / 2$, respectively. These bounds provide additional degrees of freedom to limit the probability of interference to the PUs as well as to maintain a certain level of opportunistic spectrum utilization from the SUs $\left[1-P_{q, k}^{\mathrm{fa}} \geq 1-\beta_{q, k}\right]$. Note that the constraints $\alpha_{q, k} \leq 1 / 2$ and $\beta_{q, k} \leq 1 / 2$ do not represent a real loss of generality, because practical $\mathrm{CR}$ systems are required to satisfy even stronger constraints on false alarm and detection probabilities; for instance, in the WRAN standard, $\alpha_{q, k}=\beta_{q, k}=0.1$ [33].

\subsection{Game with pricing}

We add now global interference constraints to the game theoretical formulation. In order to enforce coupling constraints while keeping the optimization as decentralized as possible, we propose the use of a pricing mechanism through a penalty in the payoff function of each player, so that the interference generated by all the SUs will depend on these prices. Prices are thus addition variables to be optimized (there is one common price associated to any of the global interference constraints); they must be chosen so that any solution of the game will satisfy the global interference constraints, which requires the introduction of additional constraints on the prices, in the form of price clearance conditions. Denoting by $\pi$ the price variable associated with the global interference constraint (8), we have the following formulation.

Player $q$ 's optimization problem is to determine, for given $\mathbf{p}_{-q}$ and $\pi$, a tuple $\left(\tau_{q}, \mathbf{p}_{q}, \boldsymbol{\gamma}_{q}\right)$ such that
\[ \operatorname{maximize}_{\tau_{q}, \mathbf{p}_{q}, \boldsymbol{\gamma}_{q}} R_{q}\left(\tau_{q}, \mathbf{p}, \gamma_{q}\right)-\pi \cdot \sum_{k=1}^{N} P_{q, k}^{\operatorname{miss}}\left(\gamma_{q, k}, \tau_{q}\right) \cdot w_{q, k} \cdot p_{q, k} \]
subject to constraints (a), (b), (c) as in (9)).
The price obeys the following complementarity condition:
\[ 0 \leq \pi \perp I^{\max }-\sum_{k=1}^{N} \sum_{q=1}^{Q} P_{q, k}^{\operatorname{miss}}\left(\gamma_{q, k}, \tau_{q}\right) \cdot w_{q, k} \cdot p_{q, k} \geq 0 . \]

In (11), the compact notation $0 \leq a \perp b \geq 0$ means $a \geq 0, b \geq 0$, and $a \cdot b=0$. The price clearance conditions (11) state that global interference constraints (8) must be satisfied together with nonnegative 
price; in addition, they imply that if the global interference constraint holds with strict inequality then the price should be zero (no penalty is needed). Thus, at any solution of the game, the optimal price is such that the interference constraint is satisfied. In the companion paper [32, we show that a pricing-based game as (10)-(11) can be solved using best-response iterative algorithms, where the price $\pi$ is updated by the SUs themselves via consensus schemes; which require a limited signaling exchange only among neighboring nodes. In Sec. 7 we show that when the SUs are willing to collaborate in the form described above, they reach higher throughputs, which motivates the formulation (10)-(11).

Note that when the price $\pi$ is set to zero, the game (10)-(11) reduces to the NEP in (9) where only the individual interference constraints (7) are imposed. Therefore, hereafter we focus only on (10)-(11), as a unified formulation including also the game (9) as special case (when $\pi=0$ ). Note that when we deal with the general formulation (10)-(11), we make the blanket assumption that none of the power/interference constraints are redundant.

\subsection{Equivalent reformulation of the games and main notation}

Before starting the analysis of the proposed games, we rewrite the players' optimization problems (10) with the side constraint (11) in a more convenient and equivalent form. The principal advantage of the reformulation is that it converts the nonlinear constraints (b) and (c) into linear constraints; thus (i) facilitating the application of the VI approach to the analysis of the game, and (ii) shedding some light on the interpretation of the new concepts and results that will be introduced later on.

At the heart of the aforementioned equivalence is a proper change of variables: instead of working with the original variables $\gamma_{q, k}$ 's and $\tau_{q}$ 's, we introduce the new variables $\widehat{\gamma}_{q, k}$ 's and $\widehat{\tau}_{q}$ 's, defined as

$$
\left(\gamma_{q, k}, \tau_{q}\right) \mapsto\left(\widehat{\gamma}_{q, k}, \widehat{\tau}_{q}\right): \quad \widehat{\tau}_{q} \triangleq \sqrt{\tau_{q} f_{q}} \quad \text { and } \quad \widehat{\gamma}_{q, k} \triangleq \sqrt{\tau_{q} f_{q}} \frac{\gamma_{q, k}-\mu_{q, k \mid 0}}{\sigma_{q, k \mid 0}}
$$

Note that the above transformation is a one-to-one mapping for any $\gamma_{q, k} \geq 0$ and $\tau_{q}>0$; we can thus reformulate the games using the new variables without loss of generality. Moreover, a property of (12) is that the constraints on $P_{q, k}^{\mathrm{fa}}\left(\gamma_{q, k}, \tau_{q}\right)$ and $P_{q, k}^{\mathrm{miss}}\left(\gamma_{q, k}, \tau_{q}\right)$ in each player's optimization problem [see (9)] become linear in the new variables $\widehat{\gamma}_{q, k}$ and $\widehat{\tau}_{q}$ : for each $k=1, \ldots, N$, we have

$$
\begin{aligned}
& P_{q, k}^{\mathrm{fa}}\left(\gamma_{q, k}, \tau_{q}\right) \leq \beta_{q, k}, \quad \Leftrightarrow \widehat{\gamma}_{q, k} \geq \mathcal{Q}^{-1}\left(\beta_{q, k}\right) \\
& P_{q, k}^{\mathrm{miss}}\left(\gamma_{q, k}, \tau_{q}\right) \leq \alpha_{q, k}, \quad \Leftrightarrow \quad \frac{\sigma_{q, k \mid 0} \widehat{\gamma}_{q, k}-\left(\mu_{q, k \mid 1}-\mu_{q, k \mid 0}\right) \widehat{\tau}_{q}}{\sigma_{q, k \mid 1}} \leq \mathcal{Q}^{-1}\left(1-\alpha_{q, k}\right),
\end{aligned}
$$

where $\mathcal{Q}^{-1}(\cdot)$ denotes the inverse of the $\mathrm{Q}$-function $[\mathcal{Q}(x)$ is a strictly decreasing function on $\mathbb{R}]$. Using the above transformation, we can equivalently rewrite the false-alarm rate $P_{q, k}^{\mathrm{fa}}\left(\gamma_{q, k}, \tau_{q}\right)$, the missed detection probability $P_{q, k}^{\text {miss }}\left(\gamma_{q, k}, \tau_{q}\right)$, and the throughput $R_{q}\left(\tau_{q}, \mathbf{p}, \gamma_{q}\right)$ of each player $q$ in terms of the new variables

$\widehat{\gamma}_{q} \triangleq\left(\widehat{\gamma}_{q, k}\right)_{k=1}^{N}$ 's and $\widehat{\tau}_{q}$ 's, denoted by $\widehat{P}_{q, k}^{\mathrm{fa}}\left(\widehat{\gamma}_{q, k}, \tau_{q}\right), \widehat{P}_{q, k}^{\text {miss }}\left(\widehat{\gamma}_{q, k}, \tau_{q}\right)$, and $\hat{R}_{q}\left(\widehat{\tau}_{q}, \mathbf{p}, \widehat{\gamma}_{q}\right)$, respectively; the explicit expression of these quantities is:

$$
P_{q, k}^{\mathrm{fa}}\left(\gamma_{q, k}, \tau_{q}\right)=\widehat{P}_{q, k}^{\mathrm{fa}}\left(\widehat{\gamma}_{q, k}\right) \triangleq \mathcal{Q}\left(\widehat{\gamma}_{q, k}\right)
$$




$$
\begin{gathered}
P_{q, k}^{\mathrm{miss}}\left(\gamma_{q, k}, \tau_{q}\right)=\widehat{P}_{q, k}^{\mathrm{miss}}\left(\widehat{\gamma}_{q, k}, \widehat{\tau}_{q}\right) \triangleq \mathcal{Q}\left(\frac{\sigma_{q, k \mid 0} \widehat{\gamma}_{q, k}-\left(\mu_{q, k \mid 1}-\mu_{q, k \mid 0}\right) \widehat{\tau}_{q}}{\sigma_{q, k \mid 1}}\right) \\
R_{q}\left(\tau_{q}, \mathbf{p}, \gamma_{q}\right)=\hat{R}_{q}\left(\widehat{\tau}_{q}, \mathbf{p}, \widehat{\gamma}_{q}\right) \triangleq\left(1-\frac{\widehat{\tau}_{q}^{2}}{f_{q} T_{q}}\right) \sum_{k=1}^{N}\left(1-\widehat{P}_{q, k}^{\mathrm{fa}}\left(\widehat{\gamma}_{q, k}, \widehat{\tau}_{q}\right)\right) r_{q, k}(\mathbf{p}) .
\end{gathered}
$$

Using (13)-(16), the game (10)-(11) in the original players' variables $\left(\tau_{q}, \mathbf{p}_{q}, \gamma_{q}\right)$ 's [and thus also (91)] can be equivalently rewritten in the new variables $\left(\hat{\tau}_{q}, \mathbf{p}_{q}, \hat{\gamma}_{q}\right)$ 's as :

Players' optimization problems. The optimization problem of player $q$ is:

$\underset{\widehat{\tau}_{q}, \mathbf{p}_{q}, \hat{\gamma}_{q}}{\operatorname{maximize}} \hat{R}_{q}\left(\widehat{\tau}_{q}, \mathbf{p}, \hat{\gamma}_{q}\right)-\pi \cdot \sum_{k=1}^{N} \widehat{P}_{q, k}^{\text {miss }}\left(\widehat{\gamma}_{q, k}, \widehat{\tau}_{q}\right) \cdot w_{q, k} \cdot p_{q, k}$

subject to

$$
\sum_{k=1}^{N} \widehat{P}_{q, k}^{\operatorname{miss}}\left(\widehat{\gamma}_{q, k}, \widehat{\tau}_{q}\right) \cdot w_{q, k} \cdot p_{q, k} \leq I_{q}^{\max }
$$

$$
\left\{\widehat{\gamma}_{q, k} \geq \widehat{\beta}_{q, k}, \quad \frac{\sigma_{q, k \mid 0} \widehat{\gamma}_{q, k}-\left(\mu_{q, k \mid 1}-\mu_{q, k \mid 0}\right) \widehat{\tau}_{q}}{\sigma_{q, k \mid 1}} \leq \widehat{\alpha}_{q, k}\right\} \quad \forall k=1, \ldots, N,
$$

$\mathbf{p}_{q} \in \mathcal{P}_{q}$ and $\widehat{\tau}_{q}^{\min } \leq \widehat{\tau}_{q} \leq \widehat{\tau}_{q}^{\max }$

Price equilibrium. The price obeys the following complementarity condition:

$$
0 \leq \pi \perp I^{\max }-\sum_{k=1}^{N} \sum_{q=1}^{Q} \widehat{P}_{q, k}^{\text {miss }}\left(\widehat{\gamma}_{q, k}, \widehat{\tau}_{q}\right) \cdot w_{q, k} \cdot p_{q, k} \geq 0
$$

where $\widehat{\alpha}_{q, k} \triangleq \mathcal{Q}^{-1}\left(1-\alpha_{q, k}\right), \widehat{\beta}_{q, k} \triangleq \mathcal{Q}^{-1}\left(\beta_{q, k}\right), \widehat{\tau}_{q}^{\min } \triangleq \sqrt{f_{q} \tau_{q}^{\min }}$, and $\widehat{\tau}_{q}^{\max } \triangleq \sqrt{f_{q} \tau_{q}^{\max }}$; and $(\widehat{a}),(\widehat{b})$, and $(\widehat{c})$ in (17) are the image under the transformation (12) of (a), (b), and (c) in (9), respectively [see (12)].

Note that (even in the transformed domain), players' optimization problems in (17) are still nonconvex, with the nonconvexity occurring in the objective function and the interference constraints $(\widehat{a})$; the constraints $(\widehat{b})$ and $(\widehat{c})$ are instead linear and thus convex; the polyhedra in $(\widehat{b})$ may be however empty. To explore such a structure, as final step, we rewrite the game (17)-(18) in a more compact form, making explicit in the feasible set of each player the polyhedral (convex) part [constraints $(\widehat{b})$ and $(\widehat{c})]$ and the nonconvex part [constraint $(\widehat{a})$ ]. Let us denote by $\mathcal{X}_{q}$ the feasible set of player $q$ in (17), given by

$$
\mathcal{X}_{q} \triangleq\left\{\left(\widehat{\tau}_{q}, \mathbf{p}_{q}, \widehat{\gamma}_{q}\right) \in \mathcal{Y}_{q} \mid I_{q}\left(\widehat{\tau}_{q}, \mathbf{p}_{q}, \widehat{\gamma}_{q}\right) \leq 0\right\}
$$

where we have separated the convex part and the nonconvex part; the convex part is given by the polyhedron $\mathcal{Y}_{q}$ corresponding to the constraints $(\widehat{b})$ and $(\widehat{c})$

$$
\mathcal{Y}_{q} \triangleq\left\{\begin{array}{cc}
\left(\widehat{\tau}_{q}, \mathbf{p}_{q}, \widehat{\gamma}_{q}\right) \mid \quad \widehat{\gamma}_{q, k} \geq \widehat{\beta}_{q, k}, \quad \frac{\sigma_{q, k \mid 0} \widehat{\gamma}_{q, k}-\left(\mu_{q, k \mid 1}-\mu_{q, k \mid 0}\right) \widehat{\tau}_{q}}{\sigma_{q, k \mid 1}} \leq \widehat{\alpha}_{q, k}, \quad \forall k=1, \ldots, N \\
\mathbf{p}_{q} \in \mathcal{P}_{q}, \quad \widehat{\tau}_{q}^{\min } \leq \widehat{\tau}_{q} \leq \widehat{\tau}_{q}^{\max }
\end{array}\right\},
$$

whereas the nonconvex part is given by the constraint $(\widehat{a})$ that we have rewritten introducing the local interference violation function 


$$
I_{q}\left(\widehat{\tau}_{q}, \mathbf{p}_{q}, \widehat{\gamma}_{q}\right) \triangleq \sum_{k=1}^{N} \widehat{P}_{q, k}^{\text {miss }}\left(\widehat{\gamma}_{q, k}, \widehat{\tau}_{q}\right) \cdot w_{q, k} \cdot p_{q, k}-I_{q}^{\max },
$$

which measure the violation of the local interference constraint $(\widehat{a})$ at $\left(\widehat{\tau}_{q}, \mathbf{p}_{q}, \widehat{\gamma}_{q}\right)$. Similarly, it is convenient to introduce also the global interference violation function $I(\widehat{\boldsymbol{\tau}}, \mathbf{p}, \widehat{\gamma})$ :

$$
I(\widehat{\boldsymbol{\tau}}, \mathbf{p}, \widehat{\gamma}) \triangleq \sum_{k=1}^{N} \sum_{q=1}^{Q} \widehat{P}_{q, k}^{\mathrm{miss}}\left(\widehat{\gamma}_{q, k}, \widehat{\tau}_{q}\right) \cdot w_{q, k} \cdot p_{q, k}-I^{\max },
$$

which measures the violation of the shared constraint at $(\widehat{\boldsymbol{\tau}}, \mathbf{p}, \widehat{\gamma})$.

Based on the above definitions, throughout the paper, we will use the following notation. The convex part of the joint strategy set is denoted by $\mathcal{Y} \triangleq \prod_{q=1}^{Q} \mathcal{Y}_{q}$, whereas the set containing all the (convex part of) players' strategy sets except the $q$-th one is denoted by $\mathcal{Y}_{-q} \triangleq \prod_{r \neq q} \mathcal{Y}_{r}$; similarly, we define $\mathcal{X} \triangleq \prod_{q=1}^{Q} \mathcal{X}_{q}$ and $\mathcal{X}_{-q} \triangleq \prod_{r \neq q} \mathcal{X}_{r}$. For notational simplicity, when it is needed, we will use interchangeably either $\left(\widehat{\boldsymbol{\tau}}_{q}, \mathbf{p}_{q}, \widehat{\boldsymbol{\gamma}}_{q}\right)$ or $\mathbf{x}_{q} \triangleq\left(\widehat{\boldsymbol{\tau}}_{q}, \mathbf{p}_{q}, \widehat{\boldsymbol{\gamma}}_{q}\right)$ to denote the strategy tuple of player $q$; similarly, $\mathbf{x} \triangleq(\widehat{\boldsymbol{\tau}}, \mathbf{p}, \widehat{\gamma})=$ $\left(\mathbf{x}_{q}\right)_{q=1}^{Q}$ will denote the strategy profile $(\widehat{\boldsymbol{\tau}}, \mathbf{p}, \widehat{\gamma})$ of all the players, with $\widehat{\boldsymbol{\tau}} \triangleq\left(\widehat{\tau}_{q}\right)_{q=1}^{Q}$, $\mathbf{p} \triangleq\left(\mathbf{p}_{q}\right)_{q=1}^{Q}$, and $\widehat{\gamma} \triangleq\left(\widehat{\gamma}_{q}\right)_{q=1}^{Q}$, whereas the strategy profile of all the players except the $q$-th one will be $\mathbf{x}_{-q} \triangleq$ $\left(\widehat{\boldsymbol{\tau}}_{r}, \mathbf{p}_{r}, \widehat{\gamma}_{r}\right)_{r=1, r \neq q}^{Q}$. Using the above definitions, game (17)-(18) can be rewritten as

Players' optimization. The optimization problem of player $q$ is:

$$
\begin{array}{ll}
\underset{\mathbf{x}_{q}}{\operatorname{maximize}} & \hat{R}_{q}\left(\mathbf{x}_{q}, \mathbf{x}_{-q}\right)-\pi \cdot I\left(\mathbf{x}_{q}, \mathbf{x}_{-q}\right) \\
\text { subject to } & \mathbf{x}_{q} \triangleq\left(\widehat{\boldsymbol{\tau}}_{q}, \mathbf{p}, \widehat{\gamma}_{q}\right) \in \mathcal{X}_{q} .
\end{array}
$$

Price equilibrium. The price obeys the following complementarity condition:

$$
0 \leq \pi \perp-I(\mathbf{x}) \geq 0 \text {. }
$$

Given the equivalence between (17)-(18) and (23)-(24), in the following we will focus on the game in the form (23)-(24) w.l.o.g.. For future convenience, Table 1 collects the main definitions and symbols used in (23) $-(24)$.

\section{Solution Analysis}

This section is devoted to the solution analysis of the game (23)-(24). It should be noted that except for the recent work [31, no existing theory to date is applicable to analyze this game. We start our analysis by studying the feasibility of each optimization problem in (23). We then introduce the definitions of NE, LNE, and the relaxed concept QNE, and establish their main properties.

\subsection{Feasibility conditions}

The feasibility of the players' problems (23) requires that all the polyhedra $\mathcal{Y}_{q}$ defined in (20) be nonempty; the following are necessary and sufficient conditions for this to hold. Introducing the SNR detection $\operatorname{snr}_{q, k}^{\mathrm{d}} \triangleq \sigma_{I_{q, k}}^{2} / \sigma_{q, k}^{2}$ experimented by the SU $q$ over carrier $k$ and using the definitions of $\sigma_{q, k \mid 1}$ and $\sigma_{q, k \mid 0}$ as given in Sec. 2.1, the feasibility requirements are: 
Table 1: Glossary of the main notation of game (23)-(24)

\begin{tabular}{|c|c|}
\hline Symbol & Meaning \\
\hline$\tau_{q}$ & sensing time of SU $q$ \\
\hline$\gamma_{q}=\left(\gamma_{q, k}\right)$ & detection thresholds of SU $q$ over carriers $k=1, \ldots, N$ \\
\hline $\mathbf{p}_{q} \triangleq\left(p_{q, k}\right)_{k=1}^{N}$ & power allocation vector of SU $q$ \\
\hline & scalar price variable \\
\hline & (transformed) sensing time of SU $q[(12)]$ \\
\hline$\widehat{\gamma}_{q}=\left(\widehat{\gamma}_{q, k}\right)$ & (transformed) detection thresholds of SU $q$ over carriers $k=1, \ldots, N[(12)]$ \\
\hline$\widehat{P}_{q, k}^{\operatorname{miss}}\left(\widehat{\tau}_{q}, \widehat{\gamma}_{q}\right)$ & (transformed) missed detection probability of SU $q$ on carrier $k$ [cf. (15)] \\
\hline $\mathbf{x}_{q} \triangleq\left(\widehat{\tau}_{q}, \mathbf{p}_{q}, \gamma_{q}\right)$ & strategy tuple of SU $q$ (in the transformed variables) \\
\hline $\begin{array}{l}\mathbf{x}_{-q} \triangleq\left(\widehat{\tau}_{r}, \mathbf{p}_{r}, \gamma_{r}\right)_{r \neq q} \\
\mathbf{x} \triangleq\left(\mathbf{x}_{q}\right)_{q=1}^{Q}=(\widehat{\boldsymbol{\tau}}, \mathbf{p}, \gamma)\end{array}$ & $\begin{array}{l}\text { strategy profile of all the SUs (in the transformed variables) except the } q \text {-th one } \\
\text { strategy profile of all the SUs (in the transformed variables) }\end{array}$ \\
\hline$\hat{R}_{q}\left(\mathbf{x}_{q}, \mathbf{x}_{-q}\right)$ & opportunistic throughput of SU $q$ in the transformed variables [cf. (16)] \\
\hline$I_{q}\left(\mathbf{x}_{q}\right)$ & local interference constraint violation of SU $q$ [cf. (21)] \\
\hline$I(\mathbf{x})$ & global interference constraint violation of SU $q$ [cf. (22)] \\
\hline $\mathcal{X}_{q}, \mathcal{X} \triangleq \prod_{q=1}^{Q} \mathcal{X}_{q}$ & feasible set of SU $q[\mathrm{cf} .(19)]$, joint feasible strategy set \\
\hline $\mathcal{X}_{-q} \triangleq \prod_{r \neq q} \mathcal{X}_{r}$ & joint strategy set of the SUs except the $q$-th one \\
\hline $\mathcal{Y}_{q}, \mathcal{Y} \triangleq \prod_{q=1}^{Q} \mathcal{Y}_{q}$ & convex part of $\mathcal{X}_{q}$ [cf. (20)], Cartesian product of all $\mathcal{Y}_{q}$ 's \\
\hline
\end{tabular}

The conditions above have an interesting physical interpretation that sheds light on the relationship between the achievable sensing performance and sensing/system parameters. It quantifies the trade-off between the sensing time (the product "time-bandwidth" $f_{q} \tau_{q}^{\max }$ of the system) and detection accuracy: the smaller the required false alarm and missed detection probabilities, the larger the number of samples and thus $\tau_{q}$ to be taken.

\subsection{NE and local NE}

The definitions of NE and local NE of a game with price equilibrium conditions as (23)-(24) are the natural generalization of the same concepts introduced for classical noncooperative games having no side constraints (see, e.g., [27, 29, 39]) and are given next.

Definition 2. A Nash equilibrium of the game (23) with side constraints (24) is a strategy-price tuple $\left(\mathbf{x}^{\star}, \pi^{\star}\right)$, such that

$$
\mathbf{x}_{q}^{\star} \in \underset{\mathbf{x}_{q} \in \mathcal{X}_{q}}{\operatorname{argmax}}\left\{\hat{R}_{q}\left(\mathbf{x}_{q}, \mathbf{x}_{-q}^{\star}\right)-\pi^{\star} \cdot I\left(\mathbf{x}_{q}, \mathbf{x}_{-q}^{\star}\right)\right\}, \quad \forall q=1, \cdots, Q
$$

and

$$
0 \leq \pi^{\star} \perp-I\left(\mathrm{x}^{\star}\right) \geq 0
$$

A local Nash equilibrium is a tuple $\left(\mathbf{x}^{\star}, \pi^{*}\right)$ for which an open neighborhood $\mathcal{N}_{q}^{\star}$ of $\mathbf{x}_{q}^{\star}$ exists such that

$$
\mathbf{x}_{q}^{\star} \in \underset{\mathbf{x}_{q} \in \mathcal{X}_{q} \cap \mathcal{N}_{q}^{\star}}{\operatorname{argmax}}\left\{\hat{R}_{q}\left(\mathbf{x}_{q}, \mathbf{x}_{-q}^{\star}\right)-\pi^{\star} \cdot I\left(\mathbf{x}_{q}, \mathbf{x}_{-q}^{\star}\right)\right\}, \quad \forall q=1, \cdots, Q
$$




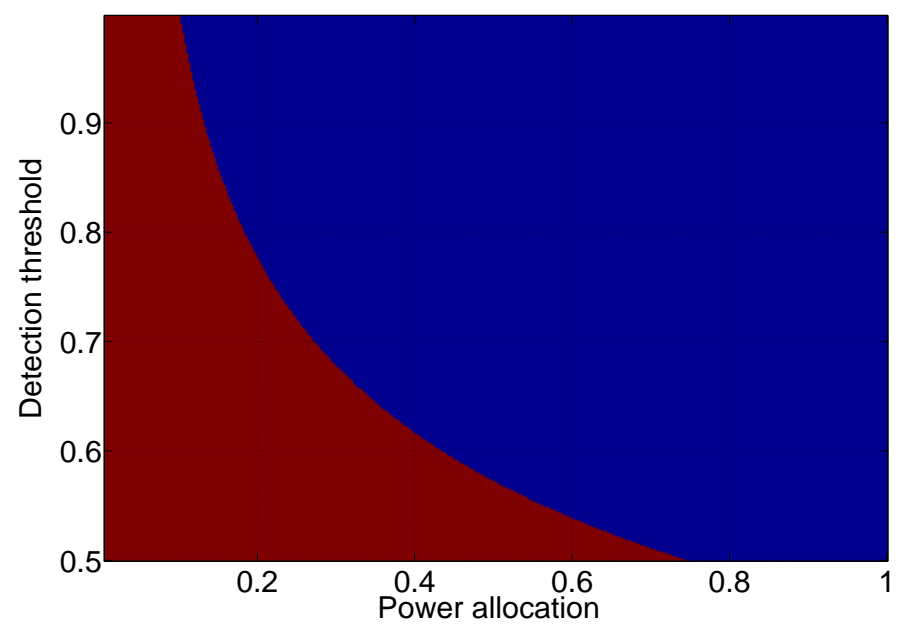

Figure 1: Example of nonconvex feasible set of a $\mathrm{SU}$ for fixed sensing time and $N=1$.

and (27) holds. A NE (LNE) is said to be trivial if $\mathbf{p}_{q}^{\star}=\mathbf{0}$ for all $q=1, \ldots, Q$.

Note that a NE is a LNE, but the converse is not necessarily true. The feasibility of the optimization problems in (23) [cf. (25)] is not enough to guarantee the existence of a (L)NE. The classical case where a NE exists is when the players' objective functions (to be maximized) are (quasi-)concave in their own variables given the other players' strategies, and the players' constraint sets are compact and convex and independent of their rivals' strategies (see, e.g., [27, 40]); other cases are when the game has a special structure, like potential or supermodular games. The game (23) with side constraints (24) has none of such properties; indeed, each player's optimization problem in (23) is nonconvex with the nonconvexity occurring in the objective function and the local/global interference constraints; moreover, the set of feasible prices [satisfying (24)] is not explicitly bounded [note that these prices cannot be normalized due to the lack of homogeneity in the players' optimization problem (23)]. Figure 1 shows an example of feasible set of the detection threshold and power allocation of a $\mathrm{SU}$, for a given sensing time and $N=1$; the set is nonconvex, implying the nonconvexity of the SUs' optimization problems in (23).

The existence of a (L)NE under the nonconvexity of the players' optimization problems and the unboundedness of the price variables is in jeopardy; in fact, the game may not even have a NE, or the NE may exist under some abstract technical conditions (that are not easy to be checked2) requiring a particular profile of the system parameters (e.g., channel conditions, interference level, SNR detection, etc...). This would make the system behavior (e.g., equilibrium existence versus nonexistence, convergence of algorithms versus nonconvergence) unpredictable, which is not a desirable feature in practical applications. To overcome this issue, in this paper, we propose the use of a (relaxed) equilibrium concept-the QNE-which will be show to alway exist (even when the NE fails). The QNE along with its main properties and optimality interpretation is introduced next.

\footnotetext{
${ }^{2}$ Conditions for the existence of a NE are established in [26, Th. 1] for NEPs (with no pricing), which may have discontinuous and/or nonquasi-concave payoff functions, but satisfying the so-called "diagonal transfer quasi-concavity" and "diagonal transfer continuity"; the case of noncompact strategy sets have been addressed in [41, Th. 5] (see also references therein). Despite their theoretical importance, the verification of these conditions for payoff functions and sets arising from realistic applications such those in this paper does not appear possible.
} 


\subsection{Quasi-Nash equilibrium}

For a nonlinear program constrained by finitely many algebraic equations and inequalities and a differentiable objective function, stationarity is defined by the well-known Karush-Kuhn-Tucker (KKT) conditions that are necessarily satisfied by a locally optimal solution under an appropriate Constraint Qualification (CQ), see [42, Prop. 1.3.4]; solutions of the KKT system are called stationary solutions of the associated optimization problem. In the context of nonconvex problems, the common approach well-accepted in practice, is then to look for a stationary (possibly locally optimal) solution. Here we apply this approach to nonconvex games and introduce the concept of QNE of the nonconvex game (23)-(24), defined as a stationary solution of the game, which is a tuple that satisfies the KKT conditions of all the players' optimization problems (23) along with the price equilibrium constraints (24). The prefix "quasi" is intended to signify that a NE (if it exists) must be a QNE under mild CQs 3 A direct study of the main properties of the QNE (e.g., existence and uniqueness) based on the KKT conditions of the game is not an easy task; thus to simplify the analysis, we rewrite first the aforementioned KKT conditions as a proper VI problem [42]; then, building on VI tools, we provide a satisfactory characterization of the QNE. It should be noted that unlike a single optimization problem where a stationary solution must exist (under a CQ) if an optimal solution exists, this is not the case for a game because a QNE is the result of concatenating the KKT conditions of several optimization problems.

At the basis of our approach there is an equivalent and nontrivial reformulation of the necessary conditions for a tuple $\left(\mathrm{x}^{\star}, \pi^{\star}\right)$ to be a (L)NE of (23)-(24), which explores the structure of the players' feasible sets $\mathcal{X}_{q}$, as described next. The classical approach to write the KKT conditions of each player's optimization problem would be introducing multipliers associated with all the constraints in the set $\mathcal{X}_{q}$-both the convex part $\mathcal{Y}_{q}$ and the nonconvex part $I_{q}\left(\mathbf{x}_{q}\right) \leq 0$ [cf. (19)] $]$-and then maximizing the resulting Lagrangian function over the whole space (i.e., considering an unconstrained optimization problem for the Lagrangian maximization). The approach we follow here is different: instead of explicitly accounting all the multipliers as variables of the KKT system, for each player's optimization problem, we introduce multipliers only for the nonconvex constraints $\left\{I_{q}\left(\mathbf{x}_{q}\right) \leq 0, q=1, \ldots, Q\right\}$ [i.e., $(\widehat{a})$ ], and retain the convex part $\mathcal{Y}_{q}$ as explicit constraints in the maximization of the resulting Lagrangian function. The mathematical formulation of this idea is given next. Denoting by $\lambda_{q}$ the multiplier associated with the nonconvex constraint $I_{q}\left(\mathbf{x}_{q}\right) \leq 0$ of player $q$, the Lagrangian function of player $q$ is:

$$
\mathcal{L}_{q}\left(\mathbf{x}, \lambda_{q}, \pi\right) \triangleq \hat{R}_{q}\left(\mathbf{x}_{q}, \mathbf{x}_{-q}\right)-\pi \cdot I\left(\mathbf{x}_{q}, \mathbf{x}_{-q}\right)-\lambda_{q} \cdot I_{q}\left(\mathbf{x}_{q}\right)
$$

which depends also on the strategies $\mathbf{x}_{-q}$ of the other players and the price $\pi$. Building on Definition 2 it is not difficult to see that if $\left(\mathbf{x}^{\star}, \pi^{\star}\right)$ is a (L)NE of (23)-(24) and some CQ holds at $\mathbf{x}^{\star}$, there exist multipliers $\boldsymbol{\lambda}^{\star} \triangleq\left(\lambda_{q}^{\star}\right)_{q=1}^{Q}$ associated with the local nonconvex constraints $\left\{I_{q}\left(\mathbf{x}_{q}\right) \leq 0, q=1, \ldots, Q\right\}$ such

\footnotetext{
${ }^{3}$ Note that without some CQs being satisfied, the KKT conditions may not be even valid necessary conditions of optimality for the optimization problems [23], making the QNE meaningless.
} 
that the tuple $\left(\mathrm{x}^{\star}, \pi^{\star}, \boldsymbol{\lambda}^{\star}\right)$ satisfies

$$
\begin{array}{cc}
\text { (i) : } & \mathbf{x}_{q}^{\star} \in \underset{\mathbf{x}_{q} \in \mathcal{Y}_{q}}{\operatorname{argmax}}\left\{\mathcal{L}_{q}\left(\mathbf{x}_{q}, \mathbf{x}_{-q}^{\star}, \lambda_{q}^{\star}, \pi^{\star}\right)\right\}, \quad \forall q=1, \cdots, Q, \\
\text { (ii) : } & 0 \leq \pi^{\star} \perp-I\left(\mathbf{x}^{\star}\right) \geq 0 \\
\text { (iii) : } & 0 \leq \lambda_{q}^{\star} \perp-I_{q}\left(\mathbf{x}_{q}^{\star}\right) \geq 0, \quad \forall q=1, \ldots, Q .
\end{array}
$$

Note that each Lagrangian maximization in (i) is constrained over the convex part $\mathcal{Y}_{q}$ of the player's local constraints $\mathcal{X}_{q}$. Since $\mathcal{Y}_{q}$ is a convex set, we can invoke the variational principle for the optimality of $\mathbf{x}_{q}^{\star}$ in (i), and obtain the following necessary conditions for (30) to hold:

$$
\begin{array}{ccc}
\text { (i') : } & \left(\mathbf{x}_{q}-\mathbf{x}_{q}^{\star}\right)^{T}\left(-\nabla_{\mathbf{x}_{q}} \mathcal{L}_{q}\left(\mathbf{x}_{q}, \mathbf{x}_{-q}^{\star}, \lambda_{q}^{\star}, \pi^{\star}\right)\right) \geq 0 \quad \forall \mathbf{x}_{q} \in \mathcal{Y}_{q} \text { and } \forall q=1, \cdots, Q, \\
\text { (ii' } \left.{ }^{\prime}\right): & \left(\pi-\pi^{\star}\right) \cdot\left(-I\left(\mathbf{x}^{\star}\right)\right) \geq 0, \quad \forall \pi \in \mathbb{R}_{+}, \\
\text {(iii } \left.{ }^{\prime}\right): & \left(\lambda_{q}-\lambda_{q}^{\star}\right) \cdot\left(-I_{q}\left(\mathbf{x}_{q}^{\star}\right)\right) \geq 0, \quad \forall \lambda_{q} \in \mathbb{R}_{+} \text {and } \forall q=1, \ldots, Q,
\end{array}
$$

where $\left(i^{\prime}\right)$ is just the aforementioned first-order optimality condition of the (nonconvex) optimization problem in (i); and (ii')-(iii') are equivalent to (ii)-(iii). Finally, since the variables in (i')-(iii') are not coupled each other by any joint constraint, we can equivalently rewrite the three separated inequalities $\left(\mathrm{i}^{\prime}\right)-\left(\mathrm{iii}^{\prime}\right)$ as one inequality, obtained just summing $\left(\mathrm{i}^{\prime}\right)-\left(\mathrm{iii}^{\prime}\right)$. More specifically, (31) is equivalent to

$$
\left(\begin{array}{c}
\mathbf{x}-\mathbf{x}^{\star} \\
\pi-\pi^{\star} \\
\boldsymbol{\lambda}-\boldsymbol{\lambda}^{\star}
\end{array}\right)^{T} \underbrace{\left(\begin{array}{c}
-\left(\nabla_{\mathbf{x}_{q}} \mathcal{L}_{q}\left(\mathbf{x}^{\star}, \pi^{\star}, \lambda_{q}^{\star},\right)\right)_{q=1}^{Q} \\
-I\left(\mathbf{x}^{\star}\right) \\
-\left(I_{q}\left(\mathbf{x}^{\star}\right)\right)_{q=1}^{Q}
\end{array}\right)}_{\triangleq \boldsymbol{\Psi}\left(\mathbf{x}^{\star}, \pi^{\star}, \boldsymbol{\lambda}^{\star}\right)} \geq 0, \quad \forall(\mathbf{x}, \pi, \boldsymbol{\lambda}) \in \underbrace{\prod_{q=1}^{Q} \mathcal{Y}_{q} \times \mathbb{R}_{+} \times \mathbb{R}_{+}^{Q}}_{\triangleq \mathcal{S}} .
$$

The above system of inequalities defines the so-called VI problem in the variables $(\mathbf{x}, \pi, \boldsymbol{\lambda})$, whose vector function is $\boldsymbol{\Psi}(\mathbf{x}, \pi, \boldsymbol{\lambda})$ and feasible set is $\mathcal{S}$, both defined in (32) 4 such a VI is denoted by $\operatorname{VI}(\mathcal{S}, \Psi)$. It follows from the implications $(30) \Rightarrow(32)$ that the $\operatorname{VI}(\mathcal{S}, \Psi)$ is an equivalent reformulation of the KKT conditions of the nonconvex game (23)-(24), wherein the convex constraints $\mathcal{Y}_{q}$ 's (and thus the associated multipliers) have been absorbed in the VI set $\mathcal{S}$. The $\operatorname{VI}(\mathcal{S}, \Psi)$ is indeed composed of three sets of variables only: i) the players' decision variables $\mathbf{x}=(\widehat{\boldsymbol{\tau}}, \mathbf{p}, \widehat{\gamma})$; ii) the multipliers $\boldsymbol{\lambda} \triangleq\left(\lambda_{q}\right)_{q=1}^{Q}$ associated with the local nonconvex constraints $\left\{I_{q}\left(\mathbf{x}_{q}\right) \leq 0, q=1, \ldots, Q\right\}$; and iii) the price $\pi$; there are no multipliers associated with the constraints $\mathcal{Y}_{q}$ 's. The above discussion is made formal in the following lemma.

Lemma 3. The KKT conditions of the game (23)-(24) are equivalent to the $\operatorname{VI}(\mathcal{S}, \mathbf{\Psi})$. The equivalence is in the following sense:

- Suppose that $\left(\mathbf{x}^{\star}, \pi^{\star}, \boldsymbol{\lambda}^{\star}, \boldsymbol{\mu}^{\star}\right)$ is a KKT solution of the game, with $\boldsymbol{\lambda}^{\star} \triangleq\left(\boldsymbol{\lambda}_{q}^{\star}\right)_{q=1}^{Q}$ and $\boldsymbol{\mu}^{\star} \triangleq\left(\boldsymbol{\mu}_{q}^{\star}\right)_{q=1}^{Q}$ being the multipliers associated with the players' nonconvex constraints $\left\{I_{q}\left(\mathbf{x}_{q}^{\star}\right) \leq 0, q=1, \ldots, Q\right\}$ and

\footnotetext{
${ }^{4}$ The $\operatorname{VI}(\mathcal{S}, \Psi)$ problem is to find a point $\mathbf{z}^{\star} \in \mathcal{S}$, the solution of the VI, such that $\left(\mathbf{z}-\mathbf{z}^{\star}\right)^{T} \boldsymbol{\Psi}\left(\mathbf{z}^{\star}\right) \geq 0$ for all $\mathbf{z} \in \mathcal{S}[42]$.
} 
convex constraints $\mathcal{Y}_{q}$ 's, respectively. Then, $\left(\mathbf{x}^{\star}, \pi^{\star}, \lambda^{\star}\right)$ is a solution of the $\operatorname{VI}(\mathcal{S}, \mathbf{\Psi})$.

- Conversely, suppose that $(\overline{\mathbf{x}}, \bar{\pi}, \overline{\boldsymbol{\lambda}})$ is a solution of the $\operatorname{VI}(\mathcal{S}, \boldsymbol{\Psi})$. Then, there exists $\overline{\boldsymbol{\mu}} \triangleq\left(\overline{\boldsymbol{\mu}}_{q}\right)_{q=1}^{Q}$ such that $(\overline{\mathbf{x}}, \bar{\pi}, \overline{\boldsymbol{\lambda}}, \overline{\boldsymbol{\mu}})$ is a KKT solution of the game, with $\overline{\boldsymbol{\mu}} \triangleq\left(\overline{\boldsymbol{\mu}}_{q}\right)_{q=1}^{Q}$ being the multipliers associated with the players' convex constraints $\mathcal{Y}_{q}$ 's.

Given the connection between a VI problem and the KKT system of the game, the QNE of the game can also be interpreted as solutions of the $\operatorname{VI}(\mathcal{S}, \Psi)$, which motivates the following formal definition of QNE of the nonconvex game (23)-(24).

Definition 4. The quasi-Nash equilibria (QNE) of the game (23) with side constraints 24) are the solutions $\left(\mathbf{x}^{\star}, \pi^{\star}, \boldsymbol{\lambda}^{\star}\right)$ of the $V I(\mathcal{S}, \Psi)$. A QNE is said to be trivial if $\mathbf{p}_{q}^{\star}=\mathbf{0}$ for all $q=1, \ldots, Q$.

Our concept of QNE is conceptually similar but formally different from other forms of local equilibria introduced in the literature, such as the critical NE [26] or the generalized equilibrium [30]. The former is indeed a feasible strategy profile of the players at which the gradients of each player's objective function (taken with respect to that player's strategy) are vanishing; the vanishing property of the gradient is typically not satisfied by solutions of constrained optimization problems, let alone equilibria of inequality constraint games. The latter equilibrium concept [30] is defined as the solution of an abstract set value inclusion, whose fruitful application to realistic games like those proposed in this paper is seriously questionable. In contrast, our VI-based definition of local equilibrium opens the way to the use of the VI machinery [42, both theory and methods, to successfully study the QNE of realistic games. The line of analysis we are going to illustrate, based on [31], is in fact a new contribution in the literature of solution analysis of local equilibria.

Optimality interpretation of the QNE. Since the QNE is a stationary solution of the game, it is desirable to understand whether the QNE has some (local) optimality properties. Interestingly, the QNE has the following equivalent interpretation: the triplet $\left(\mathbf{x}^{\star}, \pi^{\star}, \boldsymbol{\lambda}^{\star}\right)$ is a QNE of the game (23)-(24) if and only if it is an optimal solution of the players' optimization problems in the following sense [recall that $\mathbf{x}^{\star}=\left(\mathbf{x}_{q}^{\star}\right)_{q=1}^{Q}$ with $\mathbf{x}_{q}^{\star}=\left(\widehat{\tau}_{q}^{\star}, \mathbf{p}_{q}^{\star}, \widehat{\gamma}_{q}^{\star}\right)$, and $\left.\mathbf{x}_{-q}^{\star}=\left(\widehat{\tau}_{r}^{\star}, \mathbf{p}_{r}^{\star}, \widehat{\gamma}_{r}^{\star}\right)_{r \neq q}\right]$ :

(a) (NE power allocation for fixed optimal thresholds and sensing times) The tuple $\mathbf{p}^{\star}$ is a NE of the SUs' game when $\widehat{\tau}=\widehat{\tau}^{\star}$ and $\widehat{\gamma}=\widehat{\gamma}^{\star}$; for each $q=1, \cdots, Q$,

$$
\begin{aligned}
\mathbf{p}_{q}^{\star} \in \underset{\mathbf{p}_{q}}{\operatorname{argmax}}\left\{\hat{R}_{q}\left(\widehat{\tau}_{q}^{\star}, \mathbf{p}_{q}, \widehat{\gamma}_{q}^{\star}, \mathbf{x}_{-q}^{\star}\right)-\pi^{\star} \cdot I\left(\widehat{\tau}_{q}^{\star}, \mathbf{p}_{q}, \widehat{\gamma}_{q}^{\star}, \mathbf{x}_{-q}^{\star}\right)\right\} \\
\text { subject to }\left(\widehat{\tau}_{q}^{\star}, \mathbf{p}_{q}, \widehat{\gamma}_{q}^{\star}\right) \in \mathcal{X}_{q},
\end{aligned}
$$

(b) (Threshold optimization for fixed optimal power allocations and sensing times) The threshold vector $\widehat{\gamma}_{q}^{\star}$ is an optimal solution of the $q$-th players' optimization problem when $\widehat{\boldsymbol{\tau}}=\widehat{\boldsymbol{\tau}}^{\star}$ and $\mathbf{p}=\mathbf{p}^{\star}$ : for each $q=1, \cdots, Q$,

$$
\begin{aligned}
& \widehat{\gamma}_{q}^{\star} \in \underset{\widehat{\gamma}_{q}}{\operatorname{argmax}}\left\{\hat{R}_{q}\left(\widehat{\tau}_{q}^{\star}, \mathbf{p}_{q}^{\star}, \widehat{\gamma}_{q}, \mathbf{x}_{-q}^{\star}\right)-\pi^{\star} \cdot I\left(\widehat{\tau}_{q}^{\star}, \mathbf{p}_{q}^{\star}, \widehat{\gamma}_{q}, \mathbf{x}_{-q}^{\star}\right)\right\} \\
& \text { subject to }\left(\widehat{\tau}_{q}^{\star}, \mathbf{p}_{q}^{\star}, \widehat{\gamma}_{q}\right) \in \mathcal{X}_{q} ;
\end{aligned}
$$


(c) (Sensing time optimization for fixed optimal power allocations and thresholds) The sensing time $\widehat{\tau}_{q}^{\star}$ is an optimal solution of the $q$-th players' optimization problem when $\widehat{\gamma}_{q}=\widehat{\gamma}_{q}^{\star}$ and $\mathbf{p}=\mathbf{p}^{\star}$ : for each $q=1, \cdots, Q$,

$$
\begin{aligned}
\widehat{\tau}_{q}^{\star} \in \underset{\widehat{\tau}_{q}}{\operatorname{argmax}}\left\{\hat{R}_{q}\left(\widehat{\tau}_{q}, \mathbf{p}_{q}^{\star}, \widehat{\gamma}_{q}^{\star}, \mathbf{x}_{-q}^{\star}\right)-\pi^{\star} \cdot I\left(\widehat{\tau}_{q}, \mathbf{p}_{q}^{\star}, \widehat{\gamma}_{q}^{\star}, \mathbf{x}_{-q}^{\star}\right)\right\} \\
\text { subject to }\left(\widehat{\tau}_{q}, \mathbf{p}_{q}^{\star}, \widehat{\gamma}_{q}^{\star}\right) \in \mathcal{X}_{q} ;
\end{aligned}
$$

(d) (Price equilibrium) The complementarity condition $0 \leq \pi^{\star} \perp-I\left(\mathrm{x}^{\star}\right) \geq 0$ holds;

(e) (Common multipliers for individual interference constraints) For each $q=1, \cdots, Q$, there exists a common optimal multiplier tuple $\boldsymbol{\lambda}^{\star}$ associated with the individual interference constraints $\left\{I_{q}\left(\mathbf{x}_{q}^{\star}\right) \leq\right.$ $0, q=1, \ldots, Q\}$ at $\mathbf{x}^{\star}$.

In words, at a QNE $\left(\mathbf{x}^{\star}, \pi^{\star}, \boldsymbol{\lambda}^{\star}\right)$ we have that: i) each user unilaterally maximizes his own function with respect to each of his own strategies $\widehat{\tau}_{q}^{\star}, \mathbf{p}_{q}^{\star}$, and $\widehat{\gamma}_{q}^{\star}$ separately, while keeping the rivals' strategies fixed at the optimal value [statements (a)-(c) above]; ii) the optimal price value $\pi^{\star}$ satisfies the complementarity condition (24) [statement (d) above]; and iii) $\lambda^{\star}$ is the common optimal multiplier for all the players associated with the individual interference constraints in (33)-(35) [statement (e) above]. Note that (33) is a linearly constrained concave maximization problem; thus multipliers exist for this problem. Problems (34) and (35) are concave maximization program with convex constraints fulfilling the Slater CQ for a fixed but arbitrary nonzero $\mathbf{p}_{q}^{\star}$. Thus they also have constraint multipliers. Moreover, the constraint sets of problems (33), (34), and (35) are bounded. A key requirement in the QNE definition is therefore condition (e) that stipulates the existence of a set of common multiplier tuple $\boldsymbol{\lambda}^{\star}$ for the individual interference constraints, which is not automatically guaranteed. Sec. 4.5 focuses on this issue.

\subsection{Connection between LNE and QNE}

First of all, note that a $(\mathrm{L}) \mathrm{NE}\left(\mathbf{x}^{\star}, \pi^{\star}\right)$ is composed of the sensing/transmission strategies of the players as well as the price, whereas a QNE is a tuple $\left(\mathrm{x}^{\star}, \pi^{\star}, \lambda^{\star}\right)$ consisting also of the KKT multipliers $\lambda^{\star}$ of the game's nonconvex constraints $\left\{I_{q}\left(\mathbf{x}_{q}\right) \leq 0, q=1, \ldots, Q\right\}$. To explore the connection between a LNE and a QNE we need to show that, under the feasibility and solvability of all the players' optimization problems in (23) (cf. Sec. 4.1), the KKT conditions of the game are valid necessary conditions of optimality for these problems. To this end, we need to verify that an appropriate CQ holds. In this paper, we will use the Abadie's CQ (ACQ) [42, Sec. 3.2]. Not explicitly mentioned, this CQ is essentially the key to show the validity of the following result, which states that every LNE must be a QNE in this game.

Proposition 5. Given the game (23) with (possibly) side constraints 24), the following holds: if $\left(\mathbf{x}^{\star}, \pi^{\star}\right)$ is a LNE of the game then $\boldsymbol{\lambda}^{\star}$ exists such that $\left(\mathbf{x}^{\star}, \pi^{\star}, \boldsymbol{\lambda}^{\star}\right)$ is a QNE.

Note that the converse of Proposition 5 in general is not true; for a QNE to be a LNE, one needs appropriate second-order sufficiency conditions. The analysis is quite involved and goes beyond the scope of this paper; we refer the interested reader to [31, Proposition 7] for details. In the companion paper [32], we derive sufficient conditions for (a special case of) the game to have a unique QNE, which then must coincide with a $(\mathrm{L}) \mathrm{NE}$. Finally, recall that, since the set $\mathcal{S}$ of the $\operatorname{VI}(\mathcal{S}, \Psi)$ is unbounded, the existence of 
a QNE (a solution of the VI) is not automatically guaranteed. The study of existence and boundedness of the QNE is addressed in the next section.

\subsection{Existence of a QNE}

We can now study the existence of a QNE of the game (23)-(24), via the solution analysis of the VI $(\mathcal{S}, \Psi)$. The theorem below states that the game (17), if feasible, always admits a non trivial QNE.

Theorem 6. Suppose that the set $\mathcal{S}$ in (32) is nonempty [i.e., the feasibility conditions (25) hold true]. Then, the $\operatorname{VI}(\mathcal{S}, \Psi)$ has a nonempty bounded solution set; thus the game (23) with side constraints (24) has bounded QNEs. Moreover, every QNE (and thus LNE) is non trivial.

Proof. See Appendix.

Note that for the game in (9), where there are no global interference constraints (i.e., $\pi=0$ ), it is not difficult to show that every QNE (and thus LNE) is such that $\mathbf{p}_{q}^{\star} \neq \mathbf{0}$ for all $q=1, \ldots, Q$. In the presence of side constraints, instead, this is not obvious, because of the potential unboundedness of the prices $\pi$ (if the interference constraints are "too stringent", implying large prices, the users may not be allowed to transmit at all]. Interestingly, sufficient conditions for every QNE (LNE) of the game with side constraints (24) to have $\mathbf{p}_{q}^{\star} \neq \mathbf{0}$ for some/all $q=1, \ldots, Q$ can be derived (we omit the details because of the space limitation). These conditions have a physical interpretations: they impose an upper bound on the (normalized) secondary-to-primary cross-channels $\left|G_{q}(k)\right|^{2}$, so that a bounded set of equilibrium prices exists such that the interference constraints at the primary receiver can be satisfied with nonzero transmissions of the SUs.

\section{The Equi-sensing Case}

The decision model proposed so far is based on the assumption that only the PUs' signals are involved in the detection process performed by the SUs, implying that the SUs are somehow able to distinguish between primary and secondary signaling. This can be naturally accomplished if there is a common sensing time (still to optimize) during which all the SUs stay silent while sensing the spectrum. However, the joint optimization of the sensing and transmission parameters proposed in Sec. 3, in general, leads to different optimal sensing times of the SUs, implying that some SU may start transmitting while some others are still in the sensing phase. Since the energy based detection as proposed in Sec. 2.1 is not able to discriminate between sources of received energy, this in-band interference generated by the transmitting SUs would confuse the energy detector and thus introduce a significant performance degradation. To overcome this issue two different directions can be explored, as detailed next.

A first approach could be using more sophisticated signal processing techniques for the SUs' sensing that look into a primary signal footprint (e.g., modulation type, data rate, pulse shaping, or other signal feature) to improve the detector robustness, at the cost of increased complexity. This would allow the SUs to differentiate between primary signals, background noise, and interference and thus possibly benefit from adaptive signal processing for canceling SU interferers. Depending on what a priori knowledge of the primary signal is known to the SUs, different feature detectors can be applied under different scenarios and 
complexity requirements [43]. For example, ATSC digital TV signal has narrow pilot for audio and video carriers; CDMA systems have dedicated spreading codes for pilot and synchronization channels; OFDMA packets have preambles for packet acquisition. Under such a priori information, the optimal detection is given by the matched filter [36, 43]. We leave the reader the easy task to extend the proposed game theoretical formulation to the case in which the energy detector is replaced by the pilot-based matched filter (note that, under mild conditions, the performance of the matched filter are still given by (3), but with a different expression for the $\mu_{q, k \mid i}$ 's and $\sigma_{q, k \mid i}$ 's [43]). Note, however, that the better sensing performance of the matched filter are obtained at the cost of additional hardware complexity: the SUs would need a dedicated receiver for every PU class.

The second approach we propose is suitable for scenarios where feature detection is not implementable, and thus the energy detector is the only available option (see also Sec. 6] for a more general energy detectorbased scheme). In such a case, the only way for the SUs to distinguish the primary from the secondary signals is to avoid overlapping secondary transmissions during the sensing phase. This can be done by "forcing" the same sensing time for all the SUs, which still needs to be optimized. To do that while keeping the distributed nature of the optimization, we propose to modify the original games as follows.

Players' optimization. The optimization problem of player $q$ is: given $c>0$

$$
\begin{array}{ll}
\underset{\mathbf{x}_{q}}{\operatorname{maximize}} & \hat{R}_{q}\left(\mathbf{x}_{q}, \mathbf{x}_{-q}\right)-\pi \cdot I(\mathbf{x})-\frac{c}{2}\left(\frac{\hat{\tau}_{q}}{\sqrt{f_{q}}}-\frac{1}{Q} \sum_{r=1}^{Q} \frac{\hat{\tau}_{r}}{\sqrt{f_{r}}}\right)^{2} \\
\text { subject to } & \mathbf{x}_{q} \triangleq\left(\widehat{\tau}_{q}, \mathbf{p}_{q}, \widehat{\gamma}_{q}\right) \in \mathcal{X}_{q} .
\end{array}
$$

Price equilibrium. The price obeys the complementarity condition (24).

The difference with respect to the previous formulations is that now in the objective function of each player there is an additional term that works like a penalization in using different sensing times for the players. Because of this penalization, one would expect that, for sufficiently large $c$, the equilibrium of the game tends to have equal (normalized) sensing times $\hat{\tau}_{q} / \sqrt{f_{q}}$ 's (and thus equal $\tau_{q}$ 's, since $\hat{\tau}_{q} / \sqrt{f_{q}}=\sqrt{\tau_{q}}$; see (12)], provided that such a common value is feasible for all the players' optimization problems; this solution indeed is the one that minimizes the loss induced by the penalization in the payoff function of each player. This intuition is formalized in Theorem 7 below.

Feasibility conditions. The first step is to derive (sufficient) conditions guaranteeing the existence of a common value for the sensing times $\tau_{q}$ 's. We have the following: For every $\widehat{\gamma}=\left(\widehat{\gamma}_{q}\right)_{q=1}^{Q}$ for which there exist a $\mathbf{p}=\left(\mathbf{p}_{q}\right)_{q=1}^{Q}$ and $\widehat{\boldsymbol{\tau}}=\left(\widehat{\tau}_{q}\right)_{q=1}^{Q}$ satisfying the feasibility conditions $(\widehat{\mathrm{b}})$ and $(\widehat{c})$ of the original game in (17), there must exist a common $\tau$ such that, for all $q=1, \ldots, Q$ and $k=1, \ldots N$, we have

$$
\frac{\hat{\tau}_{q}^{\min }}{\sqrt{f_{q}}} \leq \sqrt{\tau} \leq \frac{\hat{\tau}_{q}^{\max }}{\sqrt{f_{q}}}, \quad \text { and } \quad \frac{\sigma_{q, k \mid 0} \widehat{\gamma}_{q, k}-\left(\mu_{q, k \mid 1}-\mu_{q, k \mid 0}\right) \sqrt{f_{q} \tau}}{\sigma_{q, k \mid 1}} \leq \widehat{\alpha}_{q, k}
$$

The first set of conditions in (37) simply postulates the existence of an overlap among the (normalized) sensing time intervals $\left[\hat{\tau}_{q}^{\min } / \sqrt{f_{q}}, \hat{\tau}_{q}^{\max } / \sqrt{f_{q}}\right]$, which is necessary to guarantee the existence of a common value for the sensing times in the original variables $\tau_{q}$ 's. The second set of conditions guarantee the 
existence of a common $\tau$ for the values of feasible $\widehat{\gamma}$ that are candidates to be QNE of the original game (17). Note that this is less than requiring $\tau$ to satisfy also the interference constraints $(\widehat{a})$ in (17) for all feasible $\widehat{\gamma}$ and $\mathbf{p}$.

Theorem 7. Given the game in (36) with side constraints (24), suppose that the feasibility conditions in (37) are satisfied. Let $\left\{c^{\nu}\right\}_{\nu=1}^{\infty}$ be any sequence of positive scalars such that $\lim _{\nu \rightarrow+\infty} c^{\nu}=+\infty$, and let $\left(\mathbf{x}^{\nu} \pi^{\nu}, \boldsymbol{\lambda}^{\nu}\right)$ be a QNE of the game for $c=c^{\nu}$. Then, the sequence $\left\{\left(\mathbf{x}^{\nu} \pi^{\nu}, \boldsymbol{\lambda}^{\nu}\right)\right\}_{\nu=1}^{\infty}$ has a limit point, denoted by $\left(\mathbf{x}^{\infty}, \pi^{\infty}, \boldsymbol{\lambda}^{\infty}\right)$, where $\mathbf{x}^{\infty} \triangleq\left(\widehat{\boldsymbol{\tau}}^{\infty}, \mathbf{p}^{\infty}, \widehat{\gamma}^{\infty}\right)$; for every such a limit point, the following hold:

(i) There exists a feasible $\tau^{\star}$ such that

$$
\frac{\hat{\tau}_{q}^{\infty}}{\sqrt{f_{q}}}=\frac{\hat{\tau}_{r}^{\infty}}{\sqrt{f_{r}}}=\sqrt{\tau^{\star}}, \quad \forall r, q=1, \ldots, Q, \text { and } r \neq q ;
$$

(ii) $\tau^{\star}$ in (38) has the following optimality properties:

$$
\begin{aligned}
\tau^{\star}=\underset{\tau}{\operatorname{argmax}} & \left\{\sum_{q=1}^{Q} \hat{R}_{q}\left(\sqrt{f_{q} \tau}, \mathbf{p}^{\infty}, \widehat{\gamma}^{\infty}\right)-\pi^{\infty} \cdot I\left(\left(\sqrt{f_{q} \tau}\right)_{q=1}^{Q}, \mathbf{p}^{\infty}, \widehat{\gamma}^{\infty}\right)\right\} \\
& \text { subject to } \quad\left(\sqrt{f_{q} \tau}, \mathbf{p}^{\infty}, \widehat{\gamma}_{q}^{\infty}\right) \in \mathcal{X}_{q}, \quad \forall q=1, \ldots, Q .
\end{aligned}
$$

Note that (38) states that in the limit all the original sensing time variables $\tau_{q}$ must be equal to $\tau^{\star}$ [recall that $\hat{\tau}_{q} / \sqrt{f_{q}}=\sqrt{\tau_{q}}$, see (12)] ], implying that there always exists a sufficiently large $c$ such that one can reach a QNE of game (36) having sensing times that differ from their average by the desired accuracy. Moreover, such a common sensing time $\tau^{\star}$ is optimal in the sense given by (39): $\tau^{\star}$ is the unique maximizer of the sum (priced) throughput of the original game (10), satisfying the price and interference constraints, while keeping the players' powers, thresholds, and price fixed to $\mathbf{p}^{\infty}, \widehat{\gamma}_{q}^{\infty}$, and $\pi^{\infty}$, respectively. In the companion paper [32], we focus on distributed algorithms to compute such limit points.

\section{Extension of the Framework}

The framework presented in this paper may be extended to cover more general settings, without affecting the validity of the obtained results. In this section, we briefly discuss some of these extensions.

Composite hypothesis testing and robust detection. The sensing model introduced in Sec. 2.1 can be generalized to the case of multiple active PUs, and the presence of device-level uncertainties (e.g., uncertainty in the power spectral density of the PUs' signals and thermal noise) as well as system level uncertainties (e.g., lack of knowledge of the number of active PUs). In this more general setting, there are $2^{P}$ configurations of possibly active PUs associated to the elements of the power set $\mathcal{P}(P)$ of $\{1, \ldots, P\} 5$ we then propose to formulate the spectrum sensing problem of the SUs as a composite hypothesis testing, based on the following two sets of hypotheses: for each $\mathrm{SU} q=1, \ldots, Q$, at carrier $k=1, \ldots, N$ and time index $n=1,2, \ldots, K_{q}$,

\footnotetext{
${ }^{5}$ The power set of a given set $\mathcal{S}$ is the set of all subsets of $\mathcal{S}$, including the empty set and $\mathcal{S}$ itself.
} 


$$
\begin{array}{lll}
\text { (PU signal absent) } \mathcal{H}_{0, k}: & y_{q, k}[n]=w_{q, k}[n] \\
\text { (PU signal present) } \mathcal{H}_{1, k}: & y_{q, k}[n]=I_{q, k \mid \mathcal{K}}[n]+w_{q, k}[n], \text { for some } \mathcal{K} \in \mathcal{P}(P) \backslash\{\emptyset\}
\end{array}
$$

where $\mathcal{H}_{0, k}$ represents the absence of any primary signal over the subcarrier $k$, and $\mathcal{H}_{1, k}$ represents the presence of (at least) one PU, with $I_{q, k \mid \mathcal{K}}[n]$ being the signal received by SU $q$ over carrier $k$ due to the presence of the active PUs, indexed by the elements of $\mathcal{K}$.

Under the assumptions that i) the noise variance over each subcarrier is known at the receiver of each SU within a given uncertainty interval; and ii) the SUs do not know the current number of active PUs out of $P$ PUs (the set $\mathcal{K}$ ), we proved that the decision rule (2), with $y_{q, k}[n]$ given in (40), is a Uniformly Most Powerful (UMP) test [35]. Roughly speaking, this means that (2) is optimal for both the false alarm and detection probabilities, in the sense that it reaches the desired false alarm rate (size of the test) while maximizing the detection probability over the entire range of noise uncertainty and uncertainty on the set of the active PUs. In [35], we showed that the (worst-case) performance of the proposed test are formally still given by (3), but with a different expression for the constants $\mu_{q, k \mid i}$ 's and $\sigma_{q, k \mid i}^{2}$ 's. Thus, the analysis developed in the previous sections apply also to this more general model; because of the space limitation, we refer to 35 ] for details.

Game-theoretical formulations in the presence of multiple PUs. To simplify the presentation, we have thus far considered scenarios where there is only one active PU and a single global interference constraint in the form of (8). The proposed game-theoretical formulations can be readily extended to the case of multiple active PUs and additional local/global interference constraints. For instance, suppose that there are (at most) $P$ active PUs along with their associated local and/or global interference constraints, e.g., given respectively by (we write the constraints directly in the transformed variables $\widehat{\gamma}_{q, k}$ and $\widehat{\tau}_{q}$ ): for each $q=1, \ldots, Q$,

$$
I_{q}^{(p)}\left(\widehat{\tau}_{q}, \mathbf{p}_{q}, \widehat{\gamma}_{q}\right) \triangleq \sum_{k=1}^{N} \widehat{P}_{q, k}^{\text {miss }}\left(\widehat{\gamma}_{q, k}, \widehat{\tau}_{q}\right) \cdot w_{q, k}^{(p)} \cdot p_{q, k}-I_{q}^{\max (p)} \leq 0, \quad p=1, \ldots, P,
$$

and

$$
I^{(p)}(\widehat{\boldsymbol{\tau}}, \mathbf{p}, \widehat{\gamma}) \triangleq \sum_{q=1}^{Q} \sum_{k=1}^{N} \widehat{P}_{q, k}^{m i s s}\left(\widehat{\gamma}_{q, k}, \widehat{\tau}_{q}\right) \cdot w_{q, k}^{(p)} \cdot p_{q, k}-I^{\max (p)} \leq 0, \quad p=1, \ldots, P,
$$

where $I_{q}^{\max }{ }^{(p)}\left[\right.$ or $\left.I^{\max (p)}\right]$ is the maximum average interference allowed to be generated by the SU $q$ [or all the SU's] that is tolerable at the primary receiver $p ; w_{q, k}^{(p)}$ 's are a given set of positive weights (possibly different for each PU $p)$; and $\widehat{P}_{q, k}^{\text {miss }}\left(\widehat{\gamma}_{q, k}, \widehat{\tau}_{q}\right)$ is the missed detection probability in the transformed variables, defined in (15). Average per-carrier local/global interference constraints can also be introduced [35].

To cast the system design in the general formulation (23)-(24), we can proceed as in Sec. 3.2. Similarly to (19) and (22), we introduce the feasible set of local constraints of each user $q$, denoted now by $\widehat{\mathcal{X}}_{q}$,

$$
\widehat{\mathcal{X}}_{q} \triangleq\left\{\left(\widehat{\tau}_{q}, \mathbf{p}_{q}, \widehat{\gamma}_{q}\right) \in \mathcal{Y}_{q} \mid I_{q}^{(p)}\left(\widehat{\tau}_{q}, \mathbf{p}_{q}, \widehat{\gamma}_{q}\right) \leq 0, \quad \forall p=1, \ldots, P\right\}
$$

with the convex part $\mathcal{Y}_{q}$ defined as in (20); and the interference violation (column) vector function 
$\mathbf{I}(\widehat{\boldsymbol{\tau}}, \mathbf{p}, \widehat{\gamma})$ grouping all the global interference constraints (42), and defined as

$$
\mathbf{I}(\widehat{\boldsymbol{\tau}}, \mathbf{p}, \widehat{\gamma}) \triangleq\left(I^{(p)}(\widehat{\boldsymbol{\tau}}, \mathbf{p}, \widehat{\gamma})\right)_{p=1}^{P}
$$

Instead of having a single price variable, in the presence of multiple global interference constraints, we associate a different price $\pi^{(p)}$ to each global interference constraint $I^{(p)}(\widehat{\boldsymbol{\tau}}, \mathbf{p}, \widehat{\gamma})$. Thus, we will have a vector of prices-the (column) vector $\boldsymbol{\pi} \triangleq\left(\pi_{p}\right)_{p=1}^{P}$-to be optimized along with multiple price clearance conditions (one for each pair price/interference constraint). Using the above notation, the resulting game theoretical formulation is the natural generalization of (23)-(24) and is given next.

Players' optimization problems. The optimization problem of player $q$ is:

$$
\begin{array}{ll}
\underset{\widehat{\tau}_{q}, \mathbf{p}_{q}, \hat{\gamma}_{q}}{\operatorname{maximize}} & \hat{R}_{q}\left(\widehat{\tau}_{q}, \mathbf{p}, \hat{\gamma}_{q}\right)-\boldsymbol{\pi}^{T} \mathbf{I}(\widehat{\boldsymbol{\tau}}, \mathbf{p}, \widehat{\gamma}) \\
\text { subject to } & \left(\widehat{\tau}_{q}, \mathbf{p}_{q}, \hat{\gamma}_{q}\right) \in \widehat{\mathcal{X}}_{q} .
\end{array}
$$

Price equilibrium. The price obeys the following complementarity conditions:

$$
0 \leq \boldsymbol{\pi} \perp-\mathbf{I}(\widehat{\boldsymbol{\tau}}, \mathbf{p}, \widehat{\gamma}) \geq \mathbf{0}
$$

The analysis of this more general game can be addressed following the same procedure as introduced in Sec. 4 for the game (23)-(24), and thus is omitted.

\section{$7 \quad$ Numerical Results}

In this section, we provide some numerical results to illustrate our theoretical findings. More specifically, we compare the performance achievable at the QNE of the proposed game with those achievable by the stateof-the-art decentralized [17] (special cases are those in [12, 13, 14]) and centralized [18, schemes proposed in the literature for similar problems; such schemes do not perform any sensing optimization using thus all the frame length for the transmission, and the QoS of the PUs is preserved by imposing (deterministic) interference constraints (we properly modified the algorithms in [18] to include the interference constraints in the feasible set of the optimization problem). Interestingly, the proposed design of CR systems based on the joint optimization of the sensing and transmission strategies is shown to outperform both centralized and decentralized current $C R$ designs, which validates our new formulations. We then show an example of the optimal sensing/throughput trade-off achievable at the QNE. Finally, we compare the sum-throughput achievable by the SUs in the presence of local and global interference constraints [game formulation (9) vs. (36) ], which sheds light on the achievable trade-off between signaling and performance.

All the numerical results here are obtained using the algorithms described in the companion paper 32. Note that some of the proposed algorithms require some signaling among the users in the form of consensus schemes; when this happens, some (finite) time of the available (sensing-transmission) frame $T$, let us say $T_{\text {cons }}$, needs to be allocated for this purpose. The interesting result is that our consensus implementation is proved to converge in a finite number of iterations ( $T_{\text {cons }}$ is then bounded). In order to 
make our numerical comparisons fair, we included this time loss in the throughput functions, which are still given by (5), but $1-\tau_{q} / T$ is replaced by $1-\left(\tau_{q}+T_{\text {cons }}\right) / T$, with $T_{\text {cons }} \leq T$ and $\tau_{q} \leq T-T_{\text {cons }}$.

Example \#1: How good is a QNE? In Figure 2 we compare the performance achievable at a QNE of the game (36) with those achievable at the stationary solutions of the sum-rate maximization problem subject to interference constraints [18] [subplot (a)] and at the NE of the game in [17] [subplot (b)]. We consider a hierarchical CR network where there are two PUs (the base stations of two cells) and fifteen SUs, randomly distributed in the cells. The (cross-)channels among the secondary links and between the primary and the secondary links are simulated as FIR filter of order $L=8$, where each tap has variance equal to $1 / L^{2}$; the available bandwidth is divided in $N=1024$ subchannels. For the sake of simplicity we consider only individual interference constraints (7), assuming the same interference limit $I^{\text {max }}$ for all SUs. Moreover we impose for each player the same false-alarm rate over all the subcarriers (possibly different among the players). To quantify the throughput gain achievable at the QNE of the proposed game, in Figure 2, we plot the (\%) ratio $\left(S R_{Q E}-S R\right) / S R$ versus the (normalized) interference constraint bound $P / I^{\max }\left(P_{q}=P_{r}=P\right.$ for all $\left.q \neq r\right)$, for different values of the SNR detection $\mathrm{snr}_{d}=\sigma_{I_{q, k}}^{2} / \sigma_{q, k}^{2}$, where $S R_{Q E}$ is the sum-throughput achievable at the QNE of the proposed game (with $c=100$ ) whereas $S R$ is either the sum-rate at a stationary solution of the social sum-rate maximization [18] subject to interference constraints [subplot (a)] or the sum-rate at the NE of the game in [17] [subplot (b)]. From the picture, we clearly see that the proposed joint optimization of the sensing and transmission parameters yields a considerable performance improvement over the current state-of-the-art CR centralized and decentralized designs, especially when the interference constraints are stringent. These results support the proposed novel formulation and concept of QNE.

Example \#2: Sensing/throughput trade-off. Figure 3 shows an example of the expected trade-off between the sensing time and the achievable throughput. More specifically, in the picture, we plot the (normalized) sum-throughput achieved at a QNE by a player of the game versus the (normalized) common sensing time, for different values of the (normalized) total interference constraint (the setup is the same as in Figure 22). According to the picture, there exists an optimal duration for the (common) sensing time at which the throughput of each SU is maximized. Moreover, as expected, more stringent interference constraints impose lower missed detection probabilities as well as false-alarm rates; requirement that is met by increasing the sensing time (i.e., making the detection more accurate). This is clear in the picture where one can see that the optimal sensing time duration increases as the interference constraints increase. In the same figure we also plot the sum-throughput achieved at the QNE of the game (36) setting $c=100$ (square markers in the plot). Interestingly, the proposed approach based on a penalty function leads to performance comparable with those achievable by a centralized approach that computes the optimal common sensing time obtained by a discrete search of such a time.

Example \#3: Global vs. local interference constraints. Global interference constraints impose less stringent conditions on the transmit power of the SUs than those imposed by the individual interference constraints, implying better throughput performance of the SUs (at the price however of more signaling among the SUs, as quantified in our companion paper [32]). Figure 4 confirms this intuition; in the 
(a): Comparison with Centralized Approaches

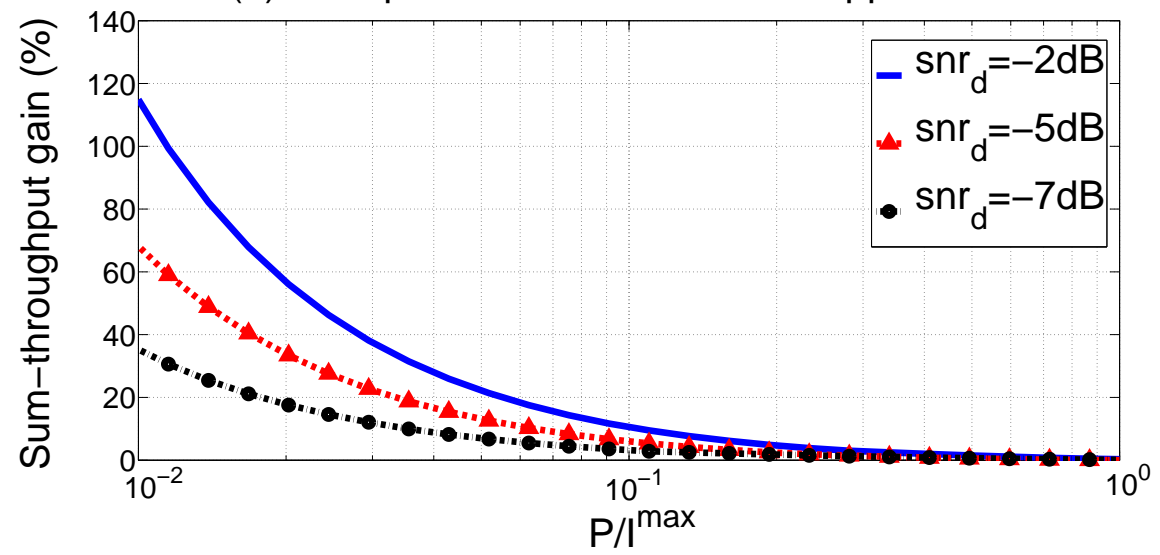

(b): Comparison with GT Approaches

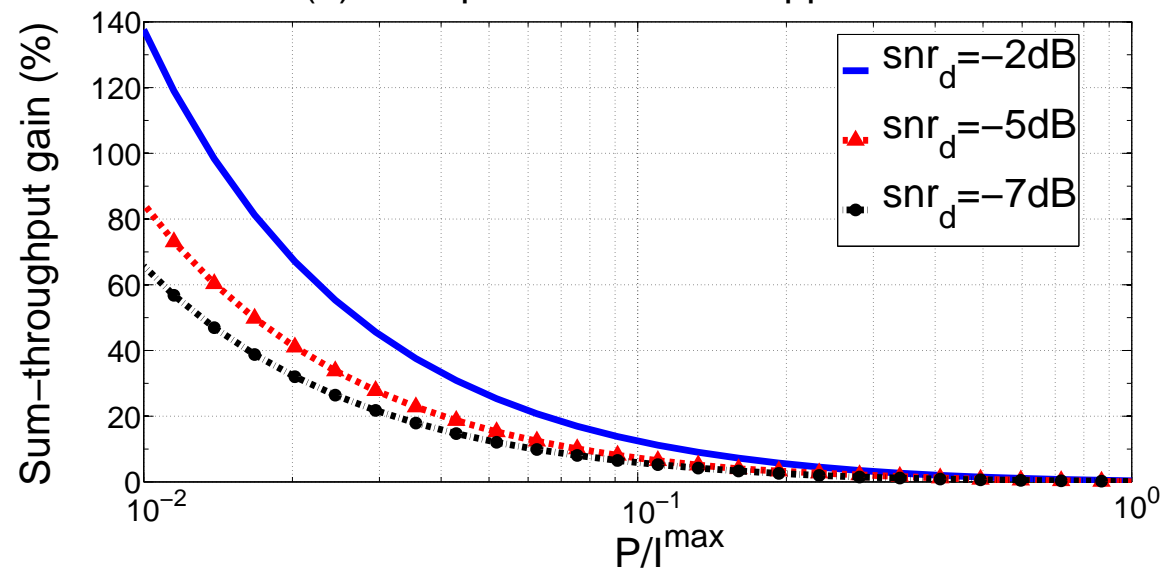

Figure 2: Performance achievable at the QNE of (36): sum-rate gain (\%) versus the normalized interference, for different values of the detection SNR and $c=100$; comparison with centralized solutions [18] [subplot (a)] and game theoretical solutions [17] [subplot (b)].

subfigure(a) we plot the average (normalized) sum-throughput of the SUs achievable in the game (9) [only local interference constraints] and (36) [global interference constraints] as a function of the maximum tolerable interference at the primary receivers, within the same setup of Figure 1 (the curves are averaged over 300 random i.i.d. Gaussian channel realizations). In (9), the interference thresholds $I_{q}^{\max }$ are set $I_{q}^{\max }=I^{\max } / Q$ for all $q$ and both PUs, so that all the SUs generate at the primary receivers the same average interference level and the aggregate average interference satisfies the imposed interference threshold $I^{\text {max }}$. In Figure $4(\mathrm{~b})$, we show an example of the average (normalized) interference profile measured at one of the (two) primary receivers, obtained solving the game (9) and (36). We clearly see from both pictures that, as expected, global interference constraints are less conservative than the local ones, yielding thus better performance of the SUs; this however comes at the price of some signaling among the SUs. 


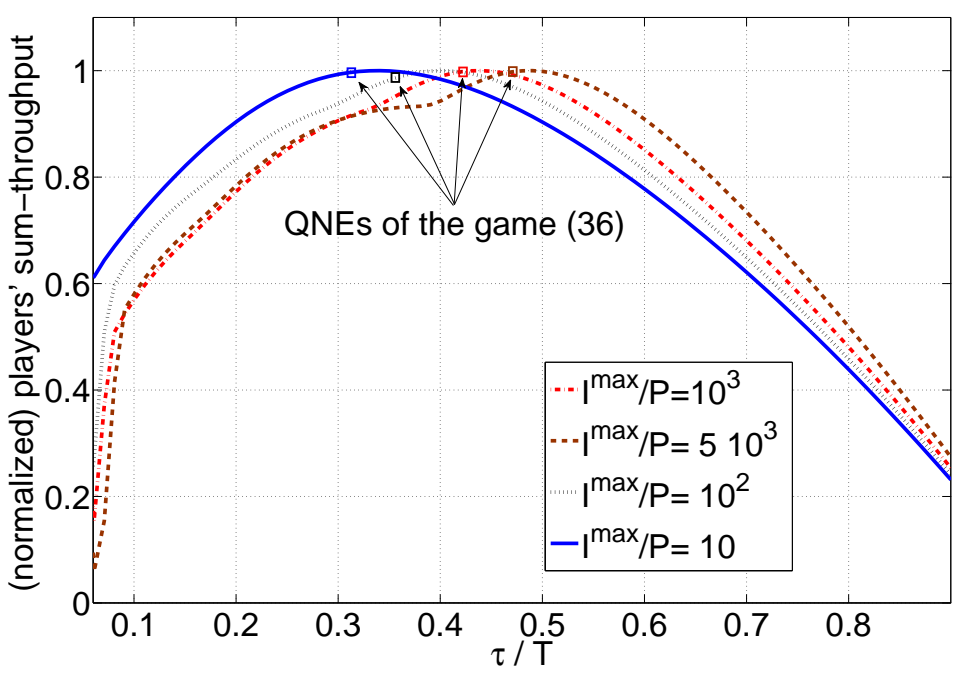

Figure 3: Example of optimal sensing/throughput trade-off: Normalized throughput versus the normalized sensing time, for different values of the (normalized) interference threshold. The square markers correspond to the QNE of the game (36), achieved with $c=100$.
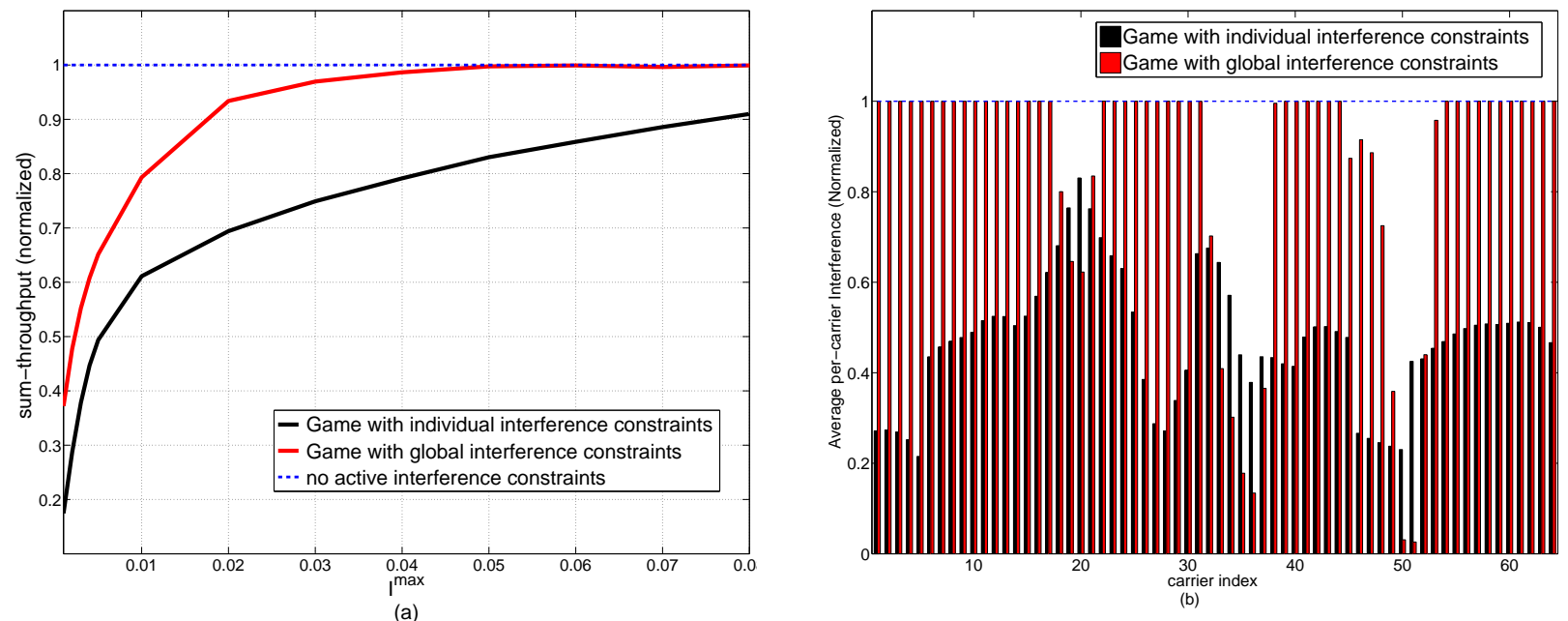

Figure 4: Global versus individual interference constraints [game (9) versus game (36)]. Subplot (a): average (normalized) sum-throughput as a function of the maximum tolerable interference $I^{\text {max }}$ at the primary receivers. Subplot (b): Average per-carrier probabilistic (normalized) interference generated by the solution of the games at one of the PU's receiver.

\section{Conclusions}

In this paper, we proposed a novel class of Nash problems wherein each SU aims to maximize his own opportunistic throughput by choosing jointly the sensing duration, the detection thresholds, and the vector power allocation over a multichannel link, under several interference constraints, either local or global. In particular, to enforce global interference constraints while keeping the optimization as decentralized as possible, we proposed a pricing mechanism that penalizes the SUs in violating the global interference 
constraints. The resulting games belong to the class of nonconvex games with unbounded pricing variables, whose analysis cannot be addressed using mathematical tools from the existing game theory literature. To deal with these issues, we proposed the use of a relaxed equilibrium concept-the QNE-and studied its properties and connection with LNE. In particular, we proved that the proposed games always have a QNE, even when a (L)NE may not exist. We then validated the QNE both theoretically and numerically: i) we proved that the QNE has some local optimality properties; and ii) numerical results show the superiority of the proposed design with respect to the state-of-the-art centralized and decentralized resource allocation algorithms for CR systems. Distributed solution schemes for computing such equilibria along with their convergence properties are proposed and studied in the companion paper 32 .

\section{Appendix: Proof of Theorem 6}

Because of the space limitation, we provide only a sketch of the proof. We need to show that the $\operatorname{VI}(\mathcal{S}, \Psi)$ in (32) has a solution. According to [42, Proposition 2.2.3], it is sufficient to find a tuple $\left(\mathbf{x}^{\text {ref }}, \pi^{\text {ref }}, \lambda^{\text {ref }}\right) \in \mathcal{S}$, with $\mathbf{x}^{\text {ref }} \triangleq\left(\widehat{\tau}_{q}^{\text {ref }}, \mathbf{p}_{q}^{\text {ref }}, \widehat{\gamma}_{q}^{\text {ref }}\right)_{q=1}^{Q}$, such that the set

$$
\mathcal{L}_{\leq} \triangleq\left\{(\mathbf{x}, \pi, \boldsymbol{\lambda}) \in \mathcal{S} \mid\left(\begin{array}{c}
\mathbf{x}-\mathrm{x}^{\mathrm{ref}} \\
\pi-\pi^{\mathrm{ref}} \\
\boldsymbol{\lambda}-\boldsymbol{\lambda}^{\mathrm{ref}}
\end{array}\right)^{T} \boldsymbol{\Psi}\left(\mathrm{x}^{\mathrm{ref}}, \pi^{\mathrm{ref}}, \boldsymbol{\lambda}^{\mathrm{ref}}\right) \leq 0\right\}
$$

is bounded. We choose $\left(\mathbf{x}^{\text {ref }}, \pi^{\text {ref }}, \boldsymbol{\lambda}^{\text {ref }}\right)$ by letting all its components to be zero except for the $\widehat{\tau}$ - and $\widehat{\gamma}$ components which we define as follows: $\widehat{\gamma}_{q, k}^{\text {ref }} \triangleq \widehat{\beta}_{q, k}$ and $\widehat{\tau}_{q}^{\text {ref }} \triangleq \widehat{\tau}_{q}^{\max }$ for all $k=1, \ldots, N$ and $q=1, \ldots, Q$. Invoking the feasibility conditions (25) and using the above definitions, we have: for all $k=1, \ldots, N$ and $q=1, \ldots, Q$,

$$
\widehat{\gamma}_{q, k}-\widehat{\gamma}_{q, k}^{\mathrm{ref}} \triangleq \frac{\sigma_{q, k \mid 0}\left(\widehat{\gamma}_{q, k}-\widehat{\beta}_{q, k}\right)-\left(\mu_{q, k \mid 1}-\mu_{q, k \mid 0}\right)\left(\widehat{\tau}_{q}-\widehat{\tau}_{q}^{\mathrm{ref}}\right)}{\sigma_{q, k \mid 1}} \geq 0 .
$$

Let $(\mathbf{x}, \pi, \boldsymbol{\lambda}) \in \mathcal{L}_{\leq}$, with the tuple $\left(\mathbf{x}^{\text {ref }}, \pi^{\text {ref }}, \boldsymbol{\lambda}^{\text {ref }}\right)$ defined above. Clearly, the players' variables $\mathbf{x}=$ $(\widehat{\boldsymbol{\tau}}, \mathbf{p}, \widehat{\gamma})$ are bounded: for every $q=1, \ldots, Q$, we have that $\left(\widehat{\boldsymbol{\tau}}_{q}, \mathbf{p}_{q}, \widehat{\boldsymbol{\gamma}}_{q}\right) \in \mathcal{X}_{q}$ implies

$\widehat{\tau}_{q}^{\max } \leq \widehat{\tau}_{q} \leq \widehat{\tau}_{q}^{\max }, \quad \mathbf{0} \leq \mathbf{p} \leq \mathbf{p}^{\max }, \quad$ and $\quad \widehat{\beta}_{q, k} \leq \widehat{\gamma}_{q, k} \leq \frac{\sigma_{q, k \mid 1} \widehat{\alpha}_{q, k}+\left(\mu_{q, k \mid 1}-\mu_{q, k \mid 0}\right) \widehat{\tau}_{q}^{\max }}{\sigma_{q, k \mid 0}} \triangleq \widehat{\gamma}_{q, k}^{\max }, \forall k$

Next, we show that the multipliers $\boldsymbol{\lambda}$ and the price $\pi$ are also bounded. Multiplying out $\left[\left(\mathbf{x}-\mathbf{x}^{\text {ref }}\right)^{T}\right.$, $\left.\left(\pi-\pi^{\text {ref }}\right)^{T},\left(\boldsymbol{\lambda}-\boldsymbol{\lambda}^{\text {ref }}\right)^{T}\right] \boldsymbol{\Psi}\left(\mathbf{x}^{\text {ref }}, \pi^{\text {ref }}, \boldsymbol{\lambda}^{\text {ref }}\right)$, using the expression of $\boldsymbol{\Psi}\left(\mathbf{x}^{\text {ref }}, \pi^{\text {ref }}, \boldsymbol{\lambda}^{\text {ref }}\right)$, canceling some 
terms, and collecting the remaining terms, we deduce

$$
\begin{aligned}
0 \geq & 2 \sum_{q=1}^{Q} \frac{\widehat{\tau}_{q}\left(\widehat{\tau}_{q}-\widehat{\tau}_{q}^{\text {ref }}\right)}{f_{q} T_{q}} \sum_{k=1}^{N}\left(1-\mathcal{Q}\left(\widehat{\gamma}_{q, k}\right)\right) r_{q, k}(\mathbf{p})+\sum_{q=1}^{Q} \sum_{k=1}^{N}\left(1-\frac{\widehat{\tau}_{q}^{2}}{f_{q} T_{q}}\right)\left(\widehat{\gamma}_{q, k}-\widehat{\beta}_{q, k}\right) \mathcal{Q}^{\prime}\left(\widehat{\gamma}_{q, k}\right) r_{q, k}(\mathbf{p}) \\
& -\sum_{q=1}^{Q} \sum_{k=1}^{N}\left(1-\frac{\widehat{\tau}_{q}^{2}}{f_{q} T_{q}}\right) \frac{\left(1-\mathcal{Q}\left(\widehat{\gamma}_{q, k}\right)\right)\left|H_{q q}(k)\right|^{2} p_{q}(k)}{\sigma_{q, k}^{2}+\sum_{r=1}^{Q}\left|H_{r q}(k)\right|^{2} p_{r}(k)}-\sum_{q=1}^{Q} \sum_{k=1}^{N}\left(\lambda_{q}+\pi\right) \cdot w_{q, k} \cdot p_{q}(k) \mathcal{Q}^{\prime}\left(\widehat{\gamma}_{q, k}\right)\left[\widehat{\gamma}_{q, k}-\widehat{\gamma}_{q, k}^{\mathrm{ref}}\right] \\
& +\sum_{q=1}^{Q} \lambda_{q} I_{q}^{\max }+\pi \cdot I^{\max } .
\end{aligned}
$$

Using the fact that the third and the fourth term on the right hand side of (49) are nonpositive [the nonpositivity of the fourth term comes from (47) and $\mathcal{Q}^{\prime}\left(\widehat{\gamma}_{q, k}\right) \leq 0$ ], we have

$$
\begin{aligned}
& \sum_{q=1}^{Q} \lambda_{q} I_{q}^{\max }+\pi \cdot I^{\max } \leq \sum_{q=1}^{Q} \sum_{k=1}^{N}\left[\left(\frac{2 \widehat{\tau}_{q}\left(\widehat{\tau}_{q}^{\text {ref }}-\widehat{\tau}_{q}\right)}{f_{q} T_{q}}\right)\left(1-\mathcal{Q}\left(\widehat{\gamma}_{q, k}\right)\right)+\left(1-\frac{\widehat{\tau}_{q}^{2}}{f_{q} T_{q}}\right)\left(\widehat{\gamma}_{q, k}-\widehat{\beta}_{q, k}\right)\left|\mathcal{Q}^{\prime}\left(\widehat{\gamma}_{q, k}\right)\right|\right] r_{q, k}(\mathbf{p}) \\
& \leq \sum_{q=1}^{Q} \sum_{k=1}^{N}\left[\frac{2 \widehat{\tau}_{q}^{\max }\left(\widehat{\tau}_{q}^{\max }-\widehat{\tau}_{q}^{\min }\right)}{f_{q} T_{q}}+\left(1-\left(\frac{\widehat{\tau}_{q}^{\min }}{\sqrt{f_{q} T_{q}}}\right)^{2}\right) \frac{\widehat{\gamma}_{q, k}^{\max }-\widehat{\beta}_{q, k}}{\sqrt{2 \pi}}\right] \log \left(1+\frac{\left|H_{q q}(k)\right|^{2} p_{q, k}^{\max }}{\sigma_{q, k}^{2}}\right)
\end{aligned}
$$

where the last inequality follows from the bounds (48) and $\left|\mathcal{Q}^{\prime}\left(\widehat{\gamma}_{q, k}\right)\right| \leq 1 / \sqrt{2 \pi}$, which establishes the boundedness of the elements in the set $\mathcal{L}_{\leq}$.

The last part of the theorem follows from Proposition 5 and the easy check that a trivial QNE cannot satisfy (32).

\section{References}

[1] J. Mitola, "Cognitive radio for flexible mobile multimedia communication," in IEEE 1999 International Workshop on Mobile Multimedia Communications (MoMuC 1999), Sandiego, California, Usa, Nov. 15-17 1999, pp. 3-10.

[2] S. Haykin, "Cognitive radio: Brain-empowered wireless communications," IEEE Jour. on Selected Areas in Communications, vol. 23, no. 2, pp. 201-220, Feb. 2005.

[3] Z. Quan, S. Cui, H. V. Poor, and A. H. Sayed, "Collaborative wideband sensing for cognitive radios: An overview of challenges and solutions," IEEE Signal Processing Magazine, vol. 25, no. 6, pp. 60-73, Nov. 2008.

[4] — , "Optimal multiband joint detection for spectrum sensing in cognitive radio networks," IEEE Trans. on Signal Processing, vol. 57, no. 3, pp. 1128-1140, March 2009.

[5] Y.-C. Liang, Y. Zeng, E. C. Y. Peh, and A. T. Hoang, "Sensing-throughput tradeoff for cognitive radio networks," IEEE Trans. on Wireless Communications, vol. 7, no. 4, pp. 1326-1337, April 2008.

[6] S.-J. Kim and G. B. Giannakis, "Rate-optimal and reduced-complexity sequential sensing algorithms for cognitive ofdm radios," EURASIP Jour., Adv. Sig. Proc., Special Issue on Dynamic Spectrum Access for Wireless Networking, vol. 2009, Sept. 2009.

[7] F. Rongfei and J. Hai, "Optimal multi-channel cooperative sensing in cognitive radio networks," IEEE Trans. on Wireless Communications, vol. 9, no. 3, pp. 1128-1138, March 2010.

[8] P. P. Hoseini and N. C. Beaulieu, "An optimal algorithm for wideband spectrum sensing in cognitive radio systems," in Proc. of the IEEE International Conference on Communications (ICC 2010), Cape Town, South Africa, May 23-27 2010. 
[9] Y. Xing, C. N. Mathur, M. Haleem, R. Chandramouli, and K. Subbalakshmi, "Dynamic spectrum access with QoS and interference temperature constraints," IEEE Trans. on Mobile Computing, vol. 6, no. 4, pp. 423-433, April 2007.

[10] Q. Lu, W. Wang, W. Wang, and T. Peng, "Asynchronous distributed power control under interference temperature constraints," in Proc. of the IEEE Global Telecommunications Conference (GLOBECOM), New Orleans, LA, USA, Nov. 30-Dec. 42008.

[11] W. Wang, T. Peng, and W. Wang, "Optimal power control under interference temperature constraints in cognitive radio network," in Proc. of the IEEE Wireless Communications and Networking Conference (WCNC 2007), Hong Kong, HK, March 11-15 2008.

[12] Z.-Q. Luo and J.-S. Pang, "Analysis of iterative waterfilling algorithm for multiuser power control in digital subscriber lines," EURASIP Jour. on Applied Signal Processing, vol. 2006, pp. 1-10, May 2006.

[13] G. Scutari, D. P. Palomar, and S. Barbarossa, "Optimal linear precoding strategies for wideband noncooperative systems based on game theory - part I\&II: Nash equilibria and distributed algorithms," IEEE Trans. on Signal Processing, vol. 56, no. 3, pp. 1230-1267, March 2008.

[14] — , "Asynchronous iterative water-filling for Gaussian frequency-selective interference channels," IEEE Trans. on Information Theory, vol. 54, no. 7, pp. 2868-2878, July 2008.

[15] A. Leshem and E. Zehavi, "Game theory and the frequency selective interference channel," IEEE Signal Processing Magazine, vol. 26, no. 5, pp. 28-40, September 2009.

[16] G. Scutari, D. Palomar, F. Facchinei, and J.-S. Pang, "Flexible design of cognitive radio wireless systems: From game theory to variational inequality theory," IEEE Signal Processing Magazine, vol. 26, no. 5, pp. 107-123, September 2009.

[17] J.-S. Pang, G. Scutari, D. P. Palomar, and F. Facchinei, "Design of cognitive radio systems under temperatureinterference constraints: A variational inequality approach," IEEE Trans. on Signal Processing, vol. 58, no. 6, pp. 3251-3271, June 2010.

[18] D. Schmidt, C. Shi, R. Berry, M. Honig, and W. Utschick, "Distributed resource allocation schemes: Pricing algorithms for power control and beamformer design in interference networks," IEEE Signal Processing Magazine, vol. 26, no. 5, pp. 53-63, Sept. 2009.

[19] E. Larsson, E. Jorswieck, J. Lindblom, and R. Mochaourab, "Game theory and the flat-fading gaussian interference channel," IEEE Signal Processing Magazine, vol. 26, no. 5, pp. 18-27, September 2009.

[20] X. Kang, Y.-C. Liang, H. K. Garg, and L. Zhang, "Sensing-based spectrum sharing in cognitive radio networks," IEEE Trans. on Vehicular Technology, vol. 58, no. 8, pp. 4649-4654, October 2009.

[21] Y. Pei, Y.-C. Liang, K. C. Teh, and K. H. Li, "How much time is needed for wideband spectrum sensing?" IEEE Trans. on Wireless Communications, vol. 8, no. 11, pp. 5466-5471, November 2008.

[22] S. Stotas and A. Nallanathan, "Optimal sensing time and power allocation in multiband cognitive radio networks," IEEE Trans. on Communications, vol. 59, no. 1, pp. 226-235, Jan. 2011.

[23] S. Barbarossa, S. Sadellitti, and G. Scutari, "Joint optimization of detection thresholds and power allocation for opportunistic access in multicarrier cognitive radio networks," in Proc. of the IEEE Third International Workshop on Computational Advances in Multi-Sensor Adaptive Processing (CAMSAP 2009), Radisson Aruba Resort, Casino \& Spa Aruba, Dutch Antilles, USA, December 13-16 2009.

[24] - "Joint optimization of detection thresholds and power allocation in multiuser wideband cognitive radios," in Proc. of Cognitive Systems with Interactive Sensors (COGIS 2009), Paris, France, Nov. 16-18 2009.

[25] X. Huang and B. Beferull-Lozano, "Non-cooperative power allocation game with imperfect sensing information for cognitive radios," in Proc. of the IEEE International Conference on Acoustics, Speech, and Signal Processing (ICASSP 2011), Prague, Czech Republic, May 22-27 2011.

[26] M. R. Baye, G. Tian, and J. Zhou, "Characterizations of the existence of equilibria in games with discontinuous and non-quasiconcave payoffs," The Review of Economic Studies, vol. 60, no. 4, pp. 935-948, Oct. 1993.

[27] J. Rosen, "Existence and uniqueness of equilibrium points for concave n-person games," Econometrica, vol. 33, no. 3, pp. 520-534, July 1965.

[28] E. van Damme, Stability and perfection of Nash equilibria. 2nd edn, Springer-Verlag, Berlin, 1996. 
[29] C. Alos-Ferrer and A. B. Ania, "Local equilibria in economic games," Economics Letters, vol. 70, no. 2, pp. 165-173, Feb. 2001.

[30] B. Cornet and M.-O. Czarnecki, "Existence of generalized equilibria," Nonlinear Analysis, vol. 44, pp. 555-574, 2001.

[31] J.-S. Pang and G. Scutari, "Nonconvex games with side constraints," SIAM Jour. on Optimization, vol. 21, no. 4, pp. 1491-1522, Dec. 2011.

[32] G. Scutari and J.-S. Pang, "Joint sensing and power allocation in nonconvex cognitive radio games: Nash equilibria and distributed algorithms," IEEE Trans. on Information Theory (to appear), 2013.

[33] Working Group on Wireless Regional Area Networks. IEEE Std. 802.22. [Online]. Available: http://www.ieee802.org/22/

[34] E. L. Lehmann, Elements of Large-Sample Theory. Springer-Verlag, New York, 1998.

[35] G. Scutari and J.-S. Pang, "Joint sensing and power allocation in nonconvex cognitive radio games: Quasi-nash equilibria," in Proc. of the 17th International Conference on Digital Signal Processing (DSP2011), Corfu, Greece, July 6-8 2011.

[36] B. C. Levy, Principles of Signal Detection and Parameter Estimation. Springer Verlag, 2008.

[37] A. Sahai, N. Hoven, and R. Tandra, "Some fundamental limits on cognitive radio," in 42rd Allerton Conf. on Communications, Control, and Computing, Allerton House, Monticello, Illinois, USA, Sept. 29-Oct. 1, 2004, pp. 1662-1671.

[38] E. Axell and E. G. Larsson, "Optimal and sub-optimal spectrum sensing of ofdm signals in known and unknown noise variance," IEEE Jour. on Selected Areas in Communications, vol. 29, no. 3, pp. 290-304, Feb. 2011.

[39] N. Schofield, "Local political equilibria," in Social Choice and Strategic Decisions, D. Austen-Smith and J. Duggan, Eds. New York: Springer, Heidelberg, 2004.

[40] F. Facchinei and J.-S. Pang, "Nash equilibria: The variational approach," in Convex Optimization in Signal Processing and Communications, D. P. Palomar and Y. C. Eldar, Eds. London: Cambridge University Press, 2009.

[41] R. Nessah and K. Kerstens, "Characterizations of the existence of nash equilibria with non-convex strategy sets," IESEG School of Management, Working Papers 2008-ECO-13, 2008. [Online]. Available: http://EconPapers.repec.org/RePEc:ies:wpaper:e200813

[42] F. Facchinei and J.-S. Pang, Finite-Dimensional Variational Inequalities and Complementarity Problem. SpringerVerlag, New York, 2003.

[43] J. Ma, G. Y. Li, and B. H. Juang, "Signal processing in cognitive radio," Proc. of the IEEE, vol. 97, no. 5, pp. 654-660, May 2009. 